\title{
9. QUATERNARY BENTHIC FORAMINIFERS FROM DEEP SEA DRILLING PROJECT SITES 612 AND 613, LEG 95, NEW JERSEY TRANSECT ${ }^{1}$
}

\author{
D. B. Scott, Centre for Marine Geology, Dalhousie University ${ }^{2}$
}

\begin{abstract}
The Quaternary benthic foraminifers from Leg 95 Sites 612 and 613 were examined with respect to paleoceanographic trends. Data from the two sites indicate the presence of markedly different bottom-water masses, during both glacial and interglacial periods. The dominant interglacial species at Site 612 is Uvigerina peregrina, which is barely present in corresponding intervals at Site 613. Dominant glacial species are Elphidium excavatum and Cassidulina reniforme at Site 612 and Epistominella takayanagii at Site 613.
\end{abstract}

\section{INTRODUCTION}

The Baltimore Canyon trough is the most intensively investigated sedimentary basin along the U.S. Atlantic margin. Offshore studies began in the 1950s (e.g., Drake et al., 1959) and have intensified as interest built because of offshore petroleum leases. On the basis of an extensive published record of both geological and geophysical investigations, the New Jersey margin was selected as the most suitable location for constructing the first marginwide stratigraphic transect.

Leg 93 started the current phase of drilling on the New Jersey transect, at two shallow sites (604 and 605) on the upper rise and one at the extreme oceanward end of the transect (603). The Leg 95 holes reported on here (612 and 613) provide the link between the shelf and the rise.

The advent of the hydraulic piston corer (HPC) has made it possible to obtain from the soft, unconsolidated Quaternary deposits high-resolution results not previously possible with rotary drilling. This chapter focuses on the benthic foraminiferal record of the Pleistocene sections at Sites 612 (1404 m water depth) and 613 (2323 m water depth). At Site 612 this includes the upper $56 \mathrm{~m}$ of sediment; at Site 613 the Pleistocene appears to be $187 \mathrm{~m}$ thick. Site 613 was cored discontinuously, and approximately $120 \mathrm{~m}$ of section was not sampled. Data will be available, however, from nearby Site 604 (Moulotte and Blanc-Vernet, in press), which was cored continuously through the entire Pleistocene section.

The purpose of this investigation is to report on Quaternary benthic foraminiferal occurrences at Sites 612 and 613 , using the assemblage characteristics to reconstruct the paleoceanography.

\section{PREVIOUS WORK}

There have been several investigations of modern faunas in this area, but most concentrated on the continen-

\footnotetext{
${ }^{1}$ Poag, C. W., Watts, A. B., et al., Init. Repts. DSDP, 95: Washington (U.S. Govt. Printing Office)

2 Address: Centre for Marine Geology, Dalhousie University, Halifax, Nova Scotia B3H 3J5 Canada.
}

tal shelf rather than the slope. Cushman (1918-1931), in his studies of the Atlantic, included localities near this area but did not include quantitative data.

The first and most comprehensive of the early quantitative studies was that by Parker (1948), who investigated four transects off the New England coast, covering water depths of 15 to $700 \mathrm{~m}$. Later studies (Murray, 1969; Gervirtz et al., 1971; Kafescioglu, 1975) employed more statistical techniques but lacked the coverage of Parker's study. More recently, Poag et al. (1980) did a comprehensive study on the shelf and upper slope off New Jersey.

Phleger (1942) examined many cores over a wide range of water depths in the same area, and his data provide a comparative base for the Holocene/Pleistocene boundary in our material.

Miller and Lohmann (1982) examined the New Jersey slope environment specifically, identifying Uvigerina peregrina as the dominant species between 1500 and $2400 \mathrm{~m}$, which agrees fairly well with what we observed at the surface of our Leg 95 sites. However, their use of a large sieve size severely limits comparison with data presented here.

Streeter (1973) and Schnitker $(1974,1979)$ examined parts of this area in connection with much wider-ranging studies.

\section{METHODS}

Ten- $\mathrm{cm}^{3}$ samples were processed for study of benthic foraminifers. All material was stored in a cold room before processing. Sediment was wet-sieved through a $63-\mu \mathrm{m}$ ( $\$ 230$ mesh) screen.

After sieving, the samples were dried and, since most had excess amounts of fine sand, the sediment was sprinkled into carbon tetrachloride to float off the foraminifers. Foraminifers were split into fractions containing 300 to 500 individuals for quantitative counts. Planktonic foraminiferal and oxygen-isotope studies on the planktonic foraminifers are in progress (D. Smith, personal communication, 1985).

A total of 72 samples from Site 612 and 50 from Site 613 were studied. Samples were obtained at larger intervals from Hole 613, both because the Quaternary section was longer and because, since it was incomplete, high-resolution samples were of less interest.

A total of 115 species were found in samples from Site 612 (Fig. 1, Table 1) and 92 species were found in samples from Site 613 (Fig. 2, Table 2). Data are reported in both absolute abundance (total numbers per $10 \mathrm{~cm}^{3}$ ) and relative frequency.

All depths given in the following sections are sub-bottom depths. 


\section{SEDIMENTOLOGY}

The sedimentology is adequately described in the site chapters (this volume), but a few brief comments are in order here. The sedimentological characteristics change little throughout the Pleistocene, except that large amounts of glauconite occur near the base of the Quaternary in both holes, particularly Hole 612 . The origin of glauconite is suggested to be shallow marine, but may be controlled more by temperature and sedimentation rate than by water depth (McRae, 1972; Bell and Goodell, 1967). Glauconite is reported not to form where water temperatures are lower than $15^{\circ} \mathrm{C}$ and/or where sedimentation rates are high (McRae, 1972). Present-day distributions confirm this (Bell and Goodell, 1967). I suggest therefore, that any glauconite occurring at Sites 612 and 613 must have been transported from upslope and that reworked materials are present when glauconite is observed.

\section{RESULTS}

\section{Hole 612}

Total numbers of individuals vary significantly throughout the section from this hole, from almost $6000 / 10 \mathrm{~cm}^{3}$ down to less than $500 / 10 \mathrm{~cm}^{3}$. A large majority of these individuals are less than $250 \mu \mathrm{m}$ and therefore were not observed in the shipboard studies (Site 612 chapter, this volume).

There are significant assemblage changes downcore (Fig. 1, Table 1). At the surface $(0-1.00 \mathrm{~m})$ a diverse fauna occurs; it is dominated by Uvigerina peregrina and Pullenia subcarinata, with lesser percentages of Epistominella exigua and Bulimina marginata. In a short sequence $(1.00-2.00 \mathrm{~m})$, the assemblage changes first to one dominated by Fursenkoina fusiformis $(1.33 \mathrm{~m})$, then to an Islandiella teretis assemblage $(1.77 \mathrm{~m})$, and below that a comparatively long $(2.00-15.00 \mathrm{~m})$ section is codominated by Elphidium excavatum f. clavata and Cassidulina reniforme. This long section is interrupted only at $4.83 \mathrm{~m}$, where an assemblage identical to the surface one reappears in one sample. This sample is suspect, since it is from the top of a core section and does not match samples from just a few centimeters above (4.48 $\mathrm{m})$ and below (5.08 m).

Below $15.00 \mathrm{~m}$, a new type of fauna begins as $E$. excavatum f. clavata and $C$. reniforme are still codominant, and there are persistent percentages of $F$. fusiformis and $I$. teretis down to $35.00 \mathrm{~m}$, but at several levels between 20.00 and $35.00 \mathrm{~m}, E$. exigua is dominant, replacing the other codominants, E. excavatum and $C$. reniforme. Below $27.00 \mathrm{~m}, I$. teretis and $F$. fusiformis replace $E$. excavatum; a peak occurrence of $F$. fusiformis at $32.00 \mathrm{~m}$ is similar to the one at $1.33 \mathrm{~m}$. In a short sequence (34.00-36.00 m), E. excavatum becomes overwhelmingly dominant. Elphidium subarcticum also appears in significant percentages in this interval.

Below the $36.00 \mathrm{~m}$ level, down to the lowest level examined in this study $(56.00 \mathrm{~m})$, the fauna again changes dramatically to an assemblage dominated by Uvigerina spp., but this assemblage is very different from the one at the surface. Most of these uvigerinids, as well as a significant part of the rest of the assemblage (e.g., Dentalina subsoluta, Bulimina aculeata), appear to be Tertiary species, but there are still significant percentages of what appear to be glacial forms (E. excavatum and $F$. fusiformis), so that I believe the Tertiary forms are reworked into the section. Using nannofossils and planktonic foraminifers, this section was described as upper Tertiary, but at least with the planktonic foraminifers, there are also significant amounts of Pleistocene forms (Poag and Low, this volume), so that older planktonic species may also be reworked from older sediments. Further evidence of reworking is the presence of glauconite layers, the top of which is at $37.00 \mathrm{~m}$. Some sedimentary structures also suggest disturbance in this section (Poag, personal communication, 1985, unpublished photos of core sections). Significantly, in the samples without glauconite ( 39.43 and $56.43 \mathrm{~m}$ ), there are no diagnostic Tertiary benthic foraminifers.

\section{Hole 613}

As mentioned, the Pleistocene section was sampled discontinuously at this site. Samples were obtained from 0 to $1.00 \mathrm{~m}, 19.00$ to $29.00 \mathrm{~m}, 59.00$ to $61.00 \mathrm{~m}, 117.00$ to $163.00 \mathrm{~m}$, and 185.00 to $187.00 \mathrm{~m}$. Total numbers are generally higher in this hole than in Hole $612(20,000 /$ $10 \mathrm{~cm}^{3}$ to $500 / 10 \mathrm{~cm}^{3}$ ); many samples contained more than $2000 / 10 \mathrm{~cm}^{3}$ (Fig. 2, Table 2). As with Hole 612, a majority of these species were less than $250 \mu \mathrm{m}$ in size and were not observed in shipboard studies (Site 613 chapter, this volume).

The surface assemblage is significantly different from that at Hole 612. U. peregrina is present, but in low percentages. The three codominant species are $I$. teretis, $F$. fusiformis, and E. excavatum f. clavata. In the next sample below the surface $(0.52 \mathrm{~m})$, however, the fauna is completely different. The dominant species becomes Epistominella takayanagii, and the other species, except $F$. fusiformis, drop off. E. subarcticum increases in relative abundance, but $U$. peregrina disappears. Total numbers decrease just below the surface as well.

There is an 18-m gap between samples, but at $19 \mathrm{~m}$ a fauna similar to that at the surface recurs and persists for a longer length of section (19-22.02 m) than at the surface. At $23.32 \mathrm{~m}$ the fauna changes to an E. takayanagii-E. subarcticum fauna (similar to that at $0.52-1.00$ m). E. takayanagii remains the dominant form down to $134.91 \mathrm{~m}$, but $E$. subarcticum diminishes starting at $26.02 \mathrm{~m}$, regains larger percentages at 59 to $61 \mathrm{~m}$, and diminishes again below that. E. excavatum f. clavata remains in high relative abundance and actually codominates with $E$. takayanagii at $28.74 \mathrm{~m}, 59.51$ to $61.01 \mathrm{~m}$, and 117.32 to $118.81 \mathrm{~m}$. After decreasing in abundance at $22.02 \mathrm{~m}, I$. teretis again becomes prominent at 117.32 $\mathrm{m}$. Also, at $120.31 \mathrm{~m}$, Bulimina marginata becomes a significant species and remains significant to $134.91 \mathrm{~m}$. F. fusiformis remains high in relative abundance (10$25 \%$ ) throughout the entire upper sections (0-134.91 m).

At the 134.91 to $136.41-\mathrm{m}$ level, E. takayanagii becomes a codominant with Brizalina subaenariensis (this is the only level in this sequence where $B$. subaenariensis 


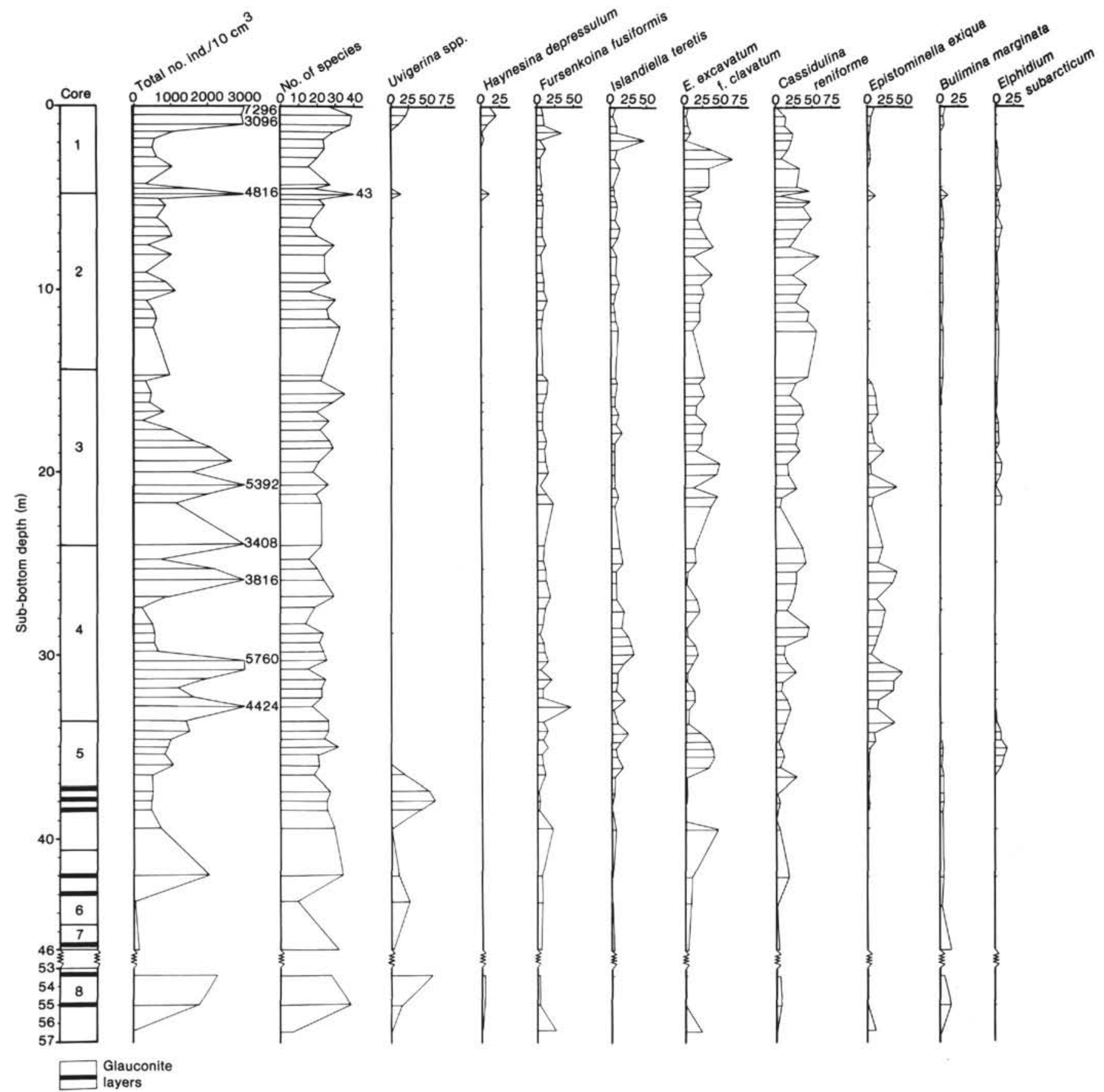

Figure 1. Percentage occurrences of selected species, Hole 612. Horizontal bars are values from corresponding level, vertical connecting lines are subjective averaging. Haynesina depressulum should be Pullenia subcarinata.

is prominent). Other species (F. fusiformis, I. teretis) drop in abundance, whereas some (B. marginata, Nonionella turigida) increase.

Just below this level, E. takayanagii recurs in overwhelming dominance $(50-70 \%)$ down to $145.93 \mathrm{~m}$. Other species all make up less than $10 \%$, but total numbers are the highest at the site $\left(5000-20,000 / 10 \mathrm{~cm}^{3}\right)$.

At $145.93 \mathrm{~m}$, the assemblage changes abruptly to one codominated by E. takayanagii, I. teretis, and E. excavatum f. clavata. This fauna persists to the bottom of the section examined here.
There appear to be reworked Tertiary-Cretaceous species throughout the hole (Table 2), but they are most prominent in the lowest sample containing glauconite $(185.23 \mathrm{~m})$, where reworked species (Heterohelix spp., Globigerinoides spp., some Dentalina spp., Guembelitria sp.) make up as much as $25 \%$ of the total fauna.

\section{BIOSTRATIGRAPHY}

There are some interpretational discrepancies between the work presented here and that performed aboard ship (Poag and Low, this volume). 


\section{B. SCOTT}

Table 1. Percentage occurrences of all benthic foraminiferal species observed in the Quaternary samples from Hole 612 .

\begin{tabular}{|c|c|c|c|c|c|c|c|c|c|c|c|c|c|c|c|c|c|}
\hline Depth of core (m) & 0.3 & 0.5 & 1.00 & 1.33 & 1.77 & 2.33 & 2.88 & 3.33 & 4.33 & 4.48 & 4.83 & 5.08 & 5.33 & 6.13 & 6.60 & 7.13 & 7.63 \\
\hline Core-Section & $1-1$ & $1-1$ & $1-1$ & $1-1$ & $1-2$ & $1-2$ & $1-2$ & $1-3$ & $1-3$ & $1-3$ & $2-1$ & $2-1$ & $2-1$ & $2-1$ & $2-2$ & $2-2$ & $2-2$ \\
\hline No. of species & 25 & 39 & 38 & 28 & 23 & 24 & 20 & 15 & 27 & 18 & 43 & 20 & 24 & 18 & 16 & 19 & 29 \\
\hline $\begin{array}{l}\text { Total number of } \\
\text { individuals } / 10 \mathrm{~cm}^{3}\end{array}$ & 7296 & 2912 & 3096 & 1034 & 536 & 508 & 590 & 1048 & 301 & 1468 & 4816 & 638 & 866 & 592 & 884 & 1028 & 339 \\
\hline $\begin{array}{l}\text { Adercotryma glomerata } \\
\text { Ammobaculites agglutinans } \\
\text { Ammonia beccarii } \\
\text { Ammotium cassis } \\
\text { Astrononion gallowayi }\end{array}$ & 1 & $\begin{array}{r}x \\
x \\
1\end{array}$ & $\mathrm{x}$ & $\mathrm{x}$ & $\mathrm{x}$ & 2 & 1 & & 1 & & $\begin{array}{r}X \\
1\end{array}$ & & $\mathrm{x}$ & & & & \\
\hline $\begin{array}{l}\text { Bolivina decussata } \\
\text { B. subspinescens } \\
\text { Brizalina bradyi } \\
\text { B. pseudopunctata } \\
\text { B. spathulata } \\
\end{array}$ & $\begin{array}{l}2 \\
4\end{array}$ & $\begin{array}{l}\mathrm{X} \\
\mathrm{X} \\
2 \\
2 \\
2\end{array}$ & $\begin{array}{r}X \\
1 \\
\\
6 \\
7\end{array}$ & $\begin{array}{l}8 \\
1\end{array}$ & 14 & $\mathrm{x}$ & 1 & 4 & ${ }^{6}$ & $\mathrm{x}^{5}$ & $\begin{array}{r}2 \\
\times \\
7\end{array}$ & $\begin{array}{l}6 \\
1 \\
\end{array}$ & $\begin{array}{l}5 \\
1\end{array}$ & $\begin{array}{l}6 \\
2\end{array}$ & 4 & $\begin{array}{l}6 \\
2\end{array}$ & $\mathbf{3}^{3}$ \\
\hline $\begin{array}{l}\text { B. subaenariensis } \\
\text { Buccella frigida } \\
\text { Bulimina aculeata } \\
\text { B. exilis } \\
\text { B. gibba } \\
\end{array}$ & & $\mathrm{x}$ & & $\mathrm{x}$ & $\mathrm{x}$ & 2 & $\begin{array}{r}\mathrm{X} \\
2\end{array}$ & 1 & 3 & $\mathrm{x}$ & $x^{1}$ & 1 & 1 & 2 & 3 & 2 & $\begin{array}{l}X \\
1\end{array}$ \\
\hline $\begin{array}{l}\text { B. marginata } \\
\text { B. striata } \\
\text { Buliminella elegantissima } \\
\text { Cassidulina laevigata } \\
\text { C. reniforme }\end{array}$ & $\mathrm{x}^{5}$ & $\begin{array}{l}2 \\
\mathrm{x}^{2} \\
14\end{array}$ & $\begin{array}{r}4 \\
1 \\
1 \\
1 \\
12\end{array}$ & $\begin{array}{c}X \\
24\end{array}$ & $\mathrm{x}$ & $\begin{array}{r}1 \\
X \\
16\end{array}$ & 8 & 31 & 1 & $\begin{array}{l}X \\
X \\
46\end{array}$ & $\begin{array}{l}8 \\
2 \\
1 \\
5\end{array}$ & $\begin{array}{l}X \\
X \\
47\end{array}$ & 36 & 2 & 1 & $\begin{array}{l}1 \\
x \\
27\end{array}$ & $\begin{array}{l}X \\
X \\
18\end{array}$ \\
\hline $\begin{array}{l}\text { Chilostomella oolina } \\
\text { Cibicides lobatulus } \\
\text { C. pseudoungerianus } \\
\text { C. robertsonianus } \\
\text { Cribrostomoides canariensis }\end{array}$ & 1 & $\mathrm{x}$ & $\begin{array}{r}X \\
5\end{array}$ & $\mathrm{x}$ & & $\mathrm{x}$ & & 1 & $\mathrm{x}$ & & $\begin{array}{r}x \\
1 \\
x\end{array}$ & & $\mathrm{x}$ & $\begin{array}{l}1 \\
1\end{array}$ & $\mathrm{x}$ & $\mathrm{x}$ & $\begin{array}{l}1 \\
2\end{array}$ \\
\hline $\begin{array}{l}\text { Cyclogyra involvens } \\
\text { Dentalina ittae } \\
\text { D. subsoluta } \\
\text { D. subemaciata } \\
\text { Dentalina spp. }\end{array}$ & & & & & & & $\mathrm{x}$ & & & & & & & & & & \\
\hline $\begin{array}{l}\text { Discorbis cf. nitida } \\
\text { Eggerella advena } \\
\text { Elphidium excavatum f. clavata } \\
\text { E. excavatum f. excavatum } \\
\text { E. excavatum f. lidoensis }\end{array}$ & 4 & 2 & $\begin{array}{l}X \\
4\end{array}$ & $\begin{array}{r}\mathrm{x} \\
\mathrm{9} \\
\mathrm{x}\end{array}$ & 1 & $\begin{array}{l}\mathrm{x} \\
37\end{array}$ & $\begin{array}{l}\mathrm{X} \\
\mathrm{X} \\
66\end{array}$ & 33 & $\begin{array}{l}x \\
34\end{array}$ & $\begin{array}{l}x \\
27\end{array}$ & 4 & 21 & $\begin{array}{l}\mathrm{X} \\
\mathrm{x} \\
22 \\
\mathrm{x}\end{array}$ & $\begin{array}{r}X \\
1 \\
17\end{array}$ & 23 & 30 & $\begin{array}{r}1 \\
39\end{array}$ \\
\hline $\begin{array}{l}\text { E. excavatum f. magna } \\
\text { E. excavatum f. selseyensis } \\
\text { E. subarcticum } \\
\text { Eoeponides pulchella } \\
\text { Eponides bradyi }\end{array}$ & 8 & $\begin{array}{l}x \\
4\end{array}$ & $\begin{array}{l}x \\
4\end{array}$ & & $\begin{array}{l}x \\
x\end{array}$ & $x^{2}$ & $\begin{array}{l}1 \\
1\end{array}$ & 2 & $\begin{array}{c}\mathrm{X} \\
7 \\
1\end{array}$ & 4 & $\begin{array}{l}x \\
x\end{array}$ & $\begin{array}{r}2 \\
x\end{array}$ & $x^{4}$ & $\begin{array}{l}1 \\
6\end{array}$ & 8 & 4 & $\begin{array}{l}5 \\
4\end{array}$ \\
\hline $\begin{array}{l}\text { E. tumidulus } \\
\text { Epistominella exigua } \\
\text { E. takayanagii } \\
\text { E. umbonifera } \\
\text { Fissurina spp. }\end{array}$ & 2 & $\begin{array}{r}4 \\
x\end{array}$ & $\begin{array}{r}3 \\
x^{2}\end{array}$ & $\begin{array}{l}1 \\
\mathrm{x} \\
\mathrm{x}\end{array}$ & $\mathrm{x}$ & $\begin{array}{l}2 \\
2\end{array}$ & 1 & $\begin{array}{l}1 \\
1\end{array}$ & $\begin{array}{l}1 \\
x\end{array}$ & $\begin{array}{r}1 \\
\mathrm{x}\end{array}$ & $\begin{array}{l}10 \\
\mathrm{X} \\
\mathrm{X}\end{array}$ & $\begin{array}{l}1 \\
1\end{array}$ & $\begin{array}{r}4 \\
\times\end{array}$ & & $\begin{array}{l}x \\
1\end{array}$ & $\begin{array}{r}4 \\
x\end{array}$ & $\begin{array}{l}x \\
x\end{array}$ \\
\hline $\begin{array}{l}\text { Frondicularia sp. } \\
\text { Fursenkoina fusiformis } \\
\text { Gavelinopsis lobatulus } \\
\text { G. translucens } \\
\text { Glabratella wrightii }\end{array}$ & 6 & 9 & $\begin{array}{l}10 \\
\mathrm{x}\end{array}$ & 35 & 3 & 12 & $\begin{array}{r}5 \\
\times\end{array}$ & 3 & 6 & 4 & $\begin{array}{l}9 \\
4\end{array}$ & 6 & 9 & 6 & $\begin{array}{r}7 \\
\times\end{array}$ & $\begin{array}{r}8 \\
\times\end{array}$ & 12 \\
\hline $\begin{array}{l}\text { Glandulina sp. } \\
\text { Globobulimina auriculata } \\
\text { Guembelitria sp. (T-K) } \\
\text { Gyroidina soldanii } \\
\text { Haynesina depressulum }\end{array}$ & $\begin{array}{r}x \\
3 \\
11\end{array}$ & $\begin{array}{r}1 \\
2 \\
20\end{array}$ & $\begin{array}{l}1 \\
4 \\
7\end{array}$ & $\begin{array}{r}2 \\
x\end{array}$ & $\begin{array}{l}1 \\
2 \\
2\end{array}$ & $\mathrm{x}$ & $\mathrm{x}$ & 2 & 1 & 2 & $\begin{array}{r}x \\
2 \\
10\end{array}$ & 1 & & $\mathrm{x}$ & $\begin{array}{l}\mathrm{X} \\
\mathrm{x}\end{array}$ & & $x$ \\
\hline $\begin{array}{l}\text { H. orbiculare } \\
\text { Heterohelix sp. (K) }\end{array}$ & & & & & & & & & $x$ & & $\mathrm{x}$ & & & & & & \\
\hline
\end{tabular}

Note: $\mathrm{X}=$ less than $1 \%$, Tertiary and Cretaceous species are indicated by $\mathrm{T}$ or $\mathrm{K}$ next to the species name.

a Haynesina depressulum should be Pullenia subcarinata.

At Site 612, the Pliocene/Pleistocene boundary is placed at $37 \mathrm{~m}$ by shipboard scientists, but I believe the boundary cannot be above my lowest sample $(56 \mathrm{~m})$. I believe this discrepancy exists because of reworked material occurring in the Pleistocene. Reworking is indicated not only by the microfossils but also by the presence of glauconite. Reworking of Tertiary and Cretaceous micro- fossils is known to occur along glacially active margins, as this area must have been during former glacial periods. In some upper Pleistocene sediments off Nova Scotia, I have found up to $90 \%$ Tertiary-Cretaceous specimens, well preserved and easily identifiable, but in clearly identifiable upper Pleistocene sediment (from a seismically continuous section; Scott, unpublished data). The 
Table 1 (continued).

\begin{tabular}{|c|c|c|c|c|c|c|c|c|c|c|c|c|c|c|c|c|c|c|c|}
\hline 8.13 & 9.13 & 9.57 & 10.13 & 10.63 & 11.07 & 11.63 & 12.13 & 14.75 & 15.03 & 15.73 & 16.25 & 16.73 & 17.23 & 17.75 & 18.27 & 18.73 & 19.37 & 19.93 & 20.73 \\
\hline $2-3$ & $2-3$ & $2-4$ & $2-4$ & $2-4$ & $2-5$ & $2-5$ & $2-5$ & $3-1$ & 3-1 & $3-1$ & $3-2$ & $3-2$ & $3-2$ & $3-3$ & $3-3$ & $3-3$ & $3-4$ & $3-4$ & 3-5 \\
\hline 24 & 24 & 27 & 15 & 30 & 25 & 26 & 32 & 23 & 21 & 35 & 28 & 19 & 26 & 21 & 26 & 28 & 21 & 17 & 26 \\
\hline 1112 & 265 & 836 & 1100 & 305 & 493 & 574 & 481 & 944 & 276 & 461 & 412 & 780 & 185 & 1016 & 1580 & 2040 & 2648 & 1488 & 5392 \\
\hline$x$ & 1 & $x$ & & $\begin{array}{l}x \\
1 \\
\end{array}$ & & $x$ & $\begin{array}{r}1 \\
x \\
x\end{array}$ & 1 & & $x$ & 1 & $x$ & 2 & & $x$ & $x$ & $\mathrm{x}$ & & 1 \\
\hline $\begin{array}{l}4 \\
1 \\
\end{array}$ & $\begin{array}{l}9 \\
1\end{array}$ & $\begin{array}{r}4 \\
\times \\
\end{array}$ & $\begin{array}{c}20 \\
\times \\
\end{array}$ & $\begin{array}{r}12 \\
1\end{array}$ & $\begin{array}{r}x \\
6 \\
x \\
\end{array}$ & $\begin{array}{r}12 \\
1 \\
\end{array}$ & $\begin{array}{r}6 \\
\times\end{array}$ & 2 & $\begin{array}{r}6 \\
\times\end{array}$ & $\begin{array}{l}x \\
x^{1}\end{array}$ & 4 & 4 & $\mathrm{x}^{2}$ & $\begin{array}{r}x \\
2\end{array}$ & $x^{3}$ & $\mathrm{x}^{4}$ & 1 & 3 & 2 \\
\hline $\begin{array}{r}X \\
1\end{array}$ & 1 & 1 & 1 & $x$ & $x$ & $x$ & 1 & $\mathrm{x}$ & 1 & 3 & 2 & & $\begin{array}{r}x \\
2\end{array}$ & 3 & 4 & $x$ & 4 & 1 & $x$ \\
\hline 59 & 24 & 1 & 32 & $\begin{array}{r}2 \\
X \\
28\end{array}$ & 46 & 43 & $\begin{array}{c}1 \\
55\end{array}$ & 43 & 27 & $\begin{array}{l}X \\
X \\
20\end{array}$ & 34 & 38 & 26 & 31 & $\begin{array}{l}x \\
x \\
26\end{array}$ & $\begin{array}{r}\mathrm{X} \\
1 \\
32 \\
\end{array}$ & $\begin{array}{c}1 \\
14\end{array}$ & $x$ & 28 \\
\hline & $\begin{array}{l}x \\
1 \\
\end{array}$ & & $x$ & 2 & 1 & & $\begin{array}{l}x \\
x \\
\end{array}$ & & & $\begin{array}{l}x \\
x\end{array}$ & & $x$ & 2 & $x$ & $x$ & $x$ & 2 & & $x$ \\
\hline & & & & & & & $\begin{array}{l}x \\
x \\
\end{array}$ & & & & & & $x$ & & & & & & $\mathrm{x}$ \\
\hline $\begin{array}{l}X \\
X \\
11\end{array}$ & $\begin{array}{r}1 \\
38\end{array}$ & $\begin{array}{c}X \\
22 \\
X\end{array}$ & 26 & $\begin{array}{c}X \\
21\end{array}$ & $\begin{array}{l}X \\
19\end{array}$ & $\begin{array}{l}X \\
X \\
20\end{array}$ & $\begin{array}{c}X X \\
X \\
10\end{array}$ & 27 & $\begin{array}{r}20 \\
1\end{array}$ & $\begin{array}{r}1 \\
1 \\
26\end{array}$ & $\begin{array}{c}1 \\
x \\
16\end{array}$ & 15 & $\begin{array}{l}X \\
30\end{array}$ & 24 & $\begin{array}{l}X \\
X \\
23\end{array}$ & $\begin{array}{l}X \\
15\end{array}$ & 47 & $\begin{array}{l}X \\
43\end{array}$ & $\begin{array}{l}X \\
10\end{array}$ \\
\hline$x$ & 2 & 3 & 1 & $\begin{array}{l}3 \\
1\end{array}$ & 3 & $\begin{array}{r}2 \\
x\end{array}$ & $\begin{array}{l}3 \\
2\end{array}$ & $\begin{array}{l}\mathrm{X} \\
2\end{array}$ & 1 & $\begin{array}{l}2 \\
2\end{array}$ & $\begin{array}{l}2 \\
x \\
x\end{array}$ & $x$ & $\begin{array}{l}3 \\
3\end{array}$ & $\begin{array}{r}3 \\
x\end{array}$ & $\begin{array}{r}5 \\
\times\end{array}$ & $\begin{array}{l}x \\
6\end{array}$ & 8 & 7 & 1 \\
\hline 3 & & $\begin{array}{r}x \\
2\end{array}$ & 1 & $x$ & 5 & $\begin{array}{r}\mathrm{X} \\
3\end{array}$ & $\begin{array}{l}X \\
X\end{array}$ & 2 & $\begin{array}{l}6 \\
1\end{array}$ & 12 & 13 & $\begin{array}{l}14 \\
X\end{array}$ & $\mathrm{x}^{5}$ & $\begin{array}{l}7 \\
2\end{array}$ & $\stackrel{9}{\mathrm{x}}$ & 22 & $\stackrel{2}{x}$ & $\stackrel{5}{x}^{5}$ & $\begin{array}{l}40 \\
X\end{array}$ \\
\hline 1 & $x$ & $x$ & & 1 & & 1 & $x$ & 1 & $x$ & & $x$ & $x$ & 1 & 1 & $x$ & $x$, & $x$ & $x$ & 1 \\
\hline $\begin{array}{r}6 \\
\times \\
\end{array}$ & 8 & $\begin{array}{r}9 \\
\times\end{array}$ & 9 & 13 & 8 & 6 & 5 & 8 & 14 & 13 & $\begin{array}{r}9 \\
\times \\
\end{array}$ & 7 & $\begin{array}{r}6 \\
\times \\
\end{array}$ & 8 & $\begin{array}{l}11 \\
\mathrm{x}\end{array}$ & 9 & 11 & 14 & $\begin{array}{r}5 \\
\times \\
\end{array}$ \\
\hline 3 & & $x$ & $x$ & $\begin{array}{c}X \\
2\end{array}$ & $\begin{array}{l}\mathrm{X} \\
\mathrm{X} \\
1 \\
\mathrm{1} \\
\mathrm{x}\end{array}$ & $\begin{array}{l}x \\
1\end{array}$ & $x$ & $\begin{array}{l}\mathrm{x} \\
\mathrm{x} \\
\mathrm{x} \\
\end{array}$ & $x$ & $\mathrm{x}$ & $\begin{array}{r}1 \\
x \\
\end{array}$ & $\begin{array}{l}\mathrm{x} \\
\mathrm{x} \\
\mathrm{x} \\
\end{array}$ & & $\begin{array}{r}x \\
1 \\
x \\
\end{array}$ & $\begin{array}{c}\mathrm{X} \\
6\end{array}$ & $\begin{array}{l}\mathrm{x} \\
1 \\
\mathrm{x} \\
\end{array}$ & 1 & $x$ & $\begin{array}{l}x \\
x \\
x\end{array}$ \\
\hline & & & & & & & & & & $x$ & & & & & & & & & \\
\hline
\end{tabular}

zonations assigned on board ship were based on nannofossils and planktonic foraminifers, but obviously the older species of these groups could have been transported together with the benthic foraminifers. It seems clear to me that more work must be done on this section to resolve the problem.

At Site $613 \mathrm{I}$ have no samples from below $186 \mathrm{~m}$, and the Pliocene/Pleistocene boundary is somewhere below that, perhaps at $190 \mathrm{~m}$ sub-bottom, according to ship- board studies. I would anticipate problems similar to those at Site 612, however, since reworked material was also found at the base of the Pleistocene at Site 613 .

\section{PALEOCEANOGRAPHY}

The benthic foraminifers do not contribute a great deal of age information, but do indicate some strong paleoceanographic signals. Although the surface assemblages at the two sites are comparable (both have Uvi- 
Table 1 (continued).

\begin{tabular}{|c|c|c|c|c|c|c|c|c|c|c|c|c|c|c|c|c|}
\hline Depth of core (m) & 21.23 & 21.73 & 24.03 & 24.83 & 25.33 & 25.87 & 26.83 & 27.37 & 28.33 & 28.83 & 29.33 & 29.83 & 30.35 & 30.83 & 31.33 & 31.83 \\
\hline Core-Section & $3-5$ & $3-5$ & 4-1 & $4-1$ & $4-1$ & $4-2$ & 4.2 & $4-3$ & $4-3$ & 4.4 & $4-4$ & $4-4$ & 4.5 & $4-5$ & 4.5 & $4-6$ \\
\hline No. of species & 19 & 22 & 22 & 15 & 19 & 23 & 29 & 18 & 13 & 24 & 21 & 23 & 25 & 14 & 24 & 22 \\
\hline $\begin{array}{l}\text { Total number of } \\
\text { individuals } / 10 \mathrm{~cm}^{3}\end{array}$ & 1992 & 1092 & 3408 & 628 & 2148 & 3816 & 890 & 176 & 472 & 557 & 548 & 662 & 5760 & 2992 & 1908 & 1136 \\
\hline $\begin{array}{l}\text { Adercotryma glomerata } \\
\text { Ammobaculites agglutinans } \\
\text { Ammonia beccarii } \\
\text { Ammotium cassis } \\
\text { Astrononion gallowayi }\end{array}$ & 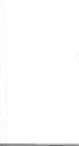 & & $\mathrm{x}$ & & $\mathrm{x}$ & $\mathrm{x}$ & $\mathrm{x}$ & $\mathrm{x}$ & & $\mathrm{x}$ & 1 & 2 & $\mathrm{x}$ & $x$ & $\mathrm{x}$ & $\mathrm{x}$ \\
\hline $\begin{array}{l}\text { Bolivina decussata } \\
\text { B. subspinescens } \\
\text { Brizalina bradyi } \\
\text { B. pseudopunctata } \\
\text { B. spathulata }\end{array}$ & $\begin{array}{r}X \\
2\end{array}$ & $\begin{array}{l}3 \\
2\end{array}$ & $\begin{array}{r}\mathrm{X} \\
3\end{array}$ & 2 & 8 & 12 & $\begin{array}{r}6 \\
x\end{array}$ & 1 & 8 & 3 & $x^{4}$ & $\begin{array}{r}5 \\
\times\end{array}$ & $\mathrm{x}$ & 1 & 2 & $\mathrm{x}$ \\
\hline $\begin{array}{l}\text { B. subaenariensis } \\
\text { Buccella frigida } \\
\text { Bulimina aculeata } \\
\text { B. exilis } \\
\text { B. gibba }\end{array}$ & $\begin{array}{r}X \\
2\end{array}$ & 4 & 1 & & $\mathrm{x}$ & $\mathrm{x}$ & $\mathrm{x}$ & 2 & & $\begin{array}{l}x \\
x\end{array}$ & 1 & & $x$ & & & \\
\hline $\begin{array}{l}\text { B. marginata } \\
\text { B. striata } \\
\text { Buliminella elegantissima } \\
\text { Cassidulina laevigata } \\
\text { C. reniforme }\end{array}$ & 6 & 5 & 36 & $\begin{array}{r}1 \\
39\end{array}$ & $\begin{array}{c}\mathrm{X} \\
28\end{array}$ & 28 & 22 & $\begin{array}{r}\mathrm{X} \\
1\end{array}$ & 46 & $\begin{array}{l}x \\
43\end{array}$ & $\begin{array}{l}7 \\
12\end{array}$ & $\begin{array}{r}\mathrm{x} \\
1 \\
11 \\
11\end{array}$ & 16 & 27 & 8 & 6 \\
\hline $\begin{array}{l}\text { Chilostomella oolina } \\
\text { Cibicides lobatulus } \\
\text { C. pseudoungerianus } \\
\text { C. robertsonianus } \\
\text { Cribrostomoides canariensis }\end{array}$ & $\mathrm{x}$ & $\mathrm{x}$ & $\mathrm{x}$ & 1 & $\mathrm{x}$ & $\mathrm{x}$ & $\mathrm{x}$ & $\mathrm{x}$ & & $\begin{array}{l}x \\
x\end{array}$ & 1 & $\mathrm{x}$ & $\mathrm{x}$ & & & $\mathrm{x}$ \\
\hline $\begin{array}{l}\text { Cyclogyra involvens } \\
\text { Dentalina ittae } \\
\text { D. subsoluta } \\
\text { D. subemaciata } \\
\text { Dentalina spp. }\end{array}$ & & & & & $\mathrm{x}$ & & & & & & & $\mathrm{x}$ & $\mathrm{x}$ & $\mathrm{x}$ & & \\
\hline $\begin{array}{l}\text { Discorbis cf. nitida } \\
\text { Eggerella advena } \\
\text { Elphidium excavatum f. clavata } \\
\text { E. excavatum f. excavatum } \\
\text { E. excavatum f. lidoensis }\end{array}$ & $\begin{array}{l}X \\
46\end{array}$ & 36 & 12 & 14 & 2 & $\underset{1}{X}$ & $\begin{array}{l}1 \\
16 \\
x\end{array}$ & 19 & $\begin{array}{l}x \\
2\end{array}$ & $x^{4}$ & 13 & $\begin{array}{r}17 \\
1\end{array}$ & $\begin{array}{l}X \\
10\end{array}$ & 3 & 2 & $\begin{array}{l}X \\
12\end{array}$ \\
\hline $\begin{array}{l}\text { E. excavatum f. magna } \\
\text { E. excavatum } \mathrm{f} \text {. selseyensis } \\
\text { E. subarcticum } \\
\text { Eoeponides pulchella } \\
\text { Eponides bradyi }\end{array}$ & 9 & $\begin{array}{r}6 \\
\times\end{array}$ & $\mathrm{x}$ & 1 & 3 & $\mathrm{x}$ & & & & $\mathrm{x}$ & $\begin{array}{r}1 \\
x\end{array}$ & & $\begin{array}{l}x \\
x\end{array}$ & $\mathrm{x}$ & $\mathrm{x}$ & 1 \\
\hline $\begin{array}{l}\text { E. tumidulus } \\
\text { Epistominella exigua } \\
\text { E. takayanagii } \\
\text { E. umbonifera } \\
\text { Fissurina spp. }\end{array}$ & $\begin{array}{r}7 \\
\times \\
\end{array}$ & $\begin{array}{l}5 \\
1\end{array}$ & 1 & 13 & 40 & $\begin{array}{l}33 \\
\mathrm{x}\end{array}$ & $\begin{array}{r}11 \\
1 \\
x \\
\end{array}$ & 24 & $\begin{array}{l}19 \\
\mathrm{X}\end{array}$ & $\begin{array}{r}13 \\
\mathrm{X} \\
1 \\
\end{array}$ & 10 & 4 & $\begin{array}{l}\mathrm{X} \\
21 \\
\mathrm{X} \\
1 \\
1\end{array}$ & 48 & $\begin{array}{l}36 \\
\mathrm{X} \\
\mathrm{X}\end{array}$ & $\begin{array}{r}35 \\
\mathrm{X} \\
1 \\
\end{array}$ \\
\hline $\begin{array}{l}\text { Frondicularia sp. } \\
\text { Fursenkoina fusiformis } \\
\text { Gavelinopsis lobatulus } \\
\text { G. translucens } \\
\text { Glabratella wrightii }\end{array}$ & 12 & 22 & 9 & 6 & 11 & 11 & 17 & 11 & 7 & 3 & 8 & $\begin{array}{l}9 \\
1\end{array}$ & 13 & 3 & 18 & 7 \\
\hline $\begin{array}{l}\text { Glandulina sp. } \\
\text { Globobulimina auriculata } \\
\text { Guembelitria sp. (T-K) } \\
\text { Gyroidina soldanii } \\
\text { Haynesina depressulum } \\
\end{array}$ & 2 & $\begin{array}{l}1 \\
1\end{array}$ & 1 & $\begin{array}{l}1 \\
1\end{array}$ & $\begin{array}{r}\mathrm{x} \\
{ }^{2} \\
\mathrm{x}\end{array}$ & 2 & $x$ & $\mathrm{x}$ & $\mathrm{x}$ & $\begin{array}{l}x \\
2\end{array}$ & $\begin{array}{r}1 \\
\mathrm{x} \\
\mathrm{x}\end{array}$ & $\mathrm{x}$ & 1 & 3 & $\begin{array}{r}3 \\
x \\
1\end{array}$ & 2 \\
\hline $\begin{array}{l}\text { H. orbiculare } \\
\text { Heterohelix sp. (K) }\end{array}$ & & & & & & & & & & & & & & & & \\
\hline
\end{tabular}

gerina peregrina), the subsurface materials differ completely. Of course, the key problem in comparing the holes is the missing upper section of Hole 613. Despite the lack of a completely comparable section, however, there was sufficient overlap to suggest a major hydrographic boundary between the two sites. $U$. peregrina occurs in both surface assemblages but dominates at Site 612 and obviously is at its lower limit at Site 613, indicating that there is a hydrographic boundary presently in this location. The surface faunas compare favorably with reported modern faunas (e.g., Miller and Lohmann, 1982 ), except that $U$. peregrina is reported to occur commonly to depths of $2400 \mathrm{~m}$.

In subsurface assemblages, however, there is less similarity. Instead of the Cassidulina reniforme-Elphidium excavatum fauna, prevalent throughout the Site 612 sequence, there is an Epistominella takayanagii fauna at Site 613 which has a strong $E$. excavatum component 
Table 1 (continued).

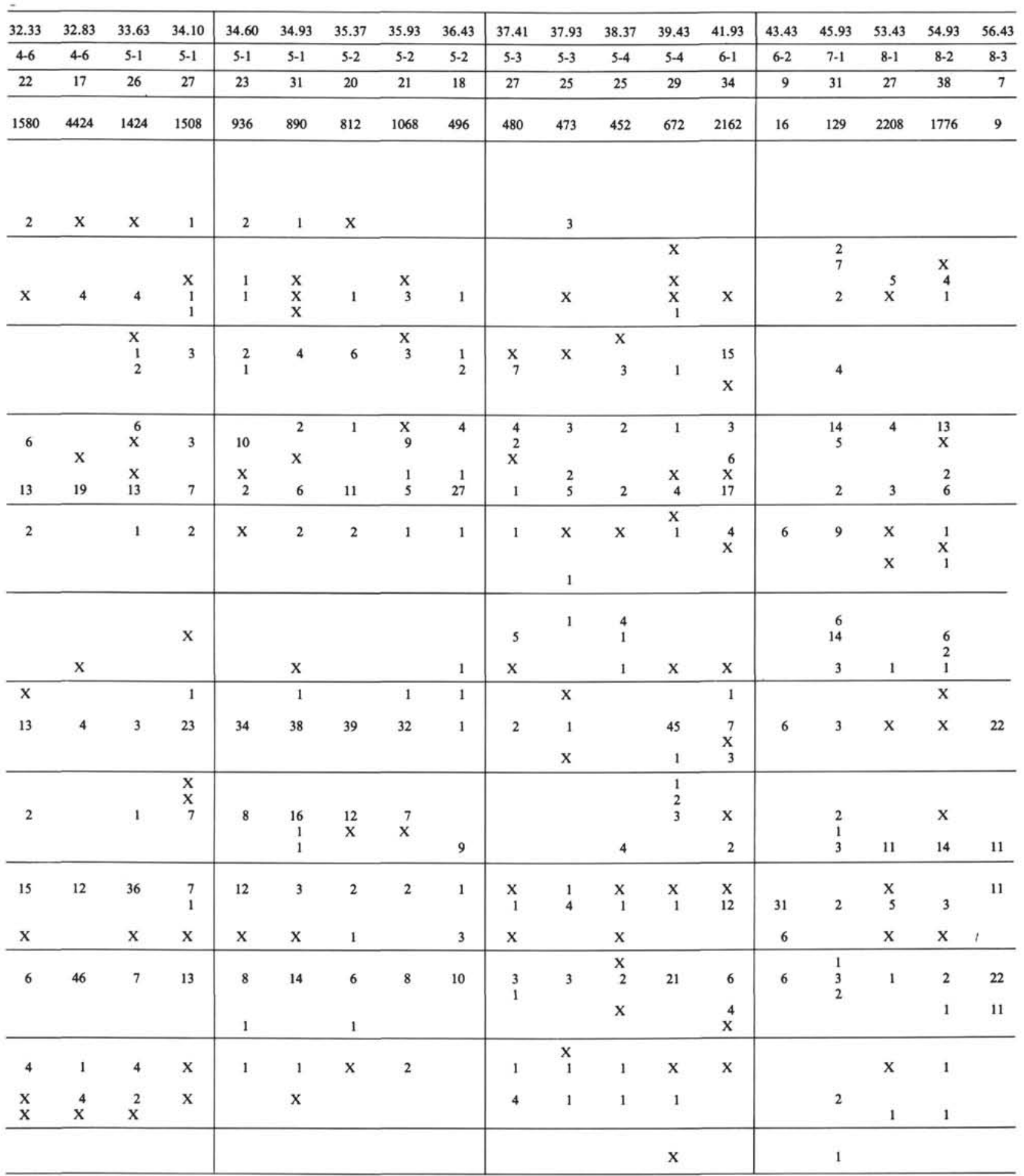

but very low relative abundances of $C$. reniforme. There also appears to be a less gradual transition between modern and glacial environments at Site 613 (i.e., surface fauna goes right into $E$. takayanagii fauna, whereas at Site 612 there are some intermediate steps).

The $C$. reniforme-E. excavatum fauna occurs very rarely in modern sediments, but has been reported in glacial marine sediments from all over the North Atlantic (e.g., Canada [Cronin, 1979; Vilks and Rashid, 1976;
Scott and Medioli, 1980; Scott et al., 1984]; Europe [Feyling-Hanssen, 1972]). Scott and Medioli (1980) and Vilks (1981) characterize the fauna as a "warm" ice-margin fauna (as opposed to present-day "cold" ice margins around Greenland, which have $100 \%$ agglutinated faunas). Most of the previous reports of this fauna are from shallow (less than $500 \mathrm{~m}$ ) water, suggesting that the $\mathrm{El}$ phidium specimens observed in slope sediments are transported. However, the work of Schafer and Cole (1982) 
D. B. SCOTT

Table 1 (continued).

\begin{tabular}{|c|c|c|c|c|c|c|c|c|c|c|c|c|c|c|c|c|c|}
\hline Depth of core (m) & 0.3 & 0.5 & 1.00 & 1.33 & 1.77 & 2.33 & 2.88 & 3.33 & 4.33 & 4.48 & 4.83 & 5.08 & 5.33 & 6.13 & 6.60 & 7.13 & 7.63 \\
\hline Core-Section & $1-1$ & $1-1$ & $1-1$ & $1-1$ & $1-2$ & $1-2$ & $1-2$ & $1-3$ & $1-3$ & $1-3$ & $2-1$ & $2-1$ & $2-1$ & $2-1$ & $2-2$ & $2-2$ & $2-2$ \\
\hline No. of species & 25 & 39 & 38 & 28 & 23 & 24 & 20 & 15 & 27 & 18 & 43 & 20 & 24 & 18 & 16 & 19 & 29 \\
\hline $\begin{array}{l}\text { Total number of } \\
\text { individuals } / 10 \mathrm{~cm}^{3}\end{array}$ & 7296 & 2912 & 3096 & 1034 & 536 & 508 & 590 & 1048 & 301 & 1468 & 4816 & 638 & 866 & 592 & 884 & 1028 & 339 \\
\hline $\begin{array}{l}\text { Hoeglundina elegans } \\
\text { Hyperammina } \mathrm{sp} . \\
\text { Islandiella teretis }\end{array}$ & $\begin{array}{c}x \\
6\end{array}$ & $\begin{array}{c}\mathrm{x} \\
2\end{array}$ & $\begin{array}{l}\mathrm{X} \\
8\end{array}$ & $\begin{array}{r}x \\
7\end{array}$ & 47 & 9 & 5 & 13 & 3 & 5 & $\begin{array}{c}\mathrm{X} \\
5\end{array}$ & 8 & 9 & 5 & 12 & 9 & 1 \\
\hline $\begin{array}{l}\text { Karreriella bradyi } \\
\text { Lagena spp. } \\
\text { Lenticulina denticulifera } \\
\text { Lenticulina spp. } \\
\text { Miliolinella subrotunda }\end{array}$ & $\mathrm{x}$ & $\begin{array}{r}1 \\
x\end{array}$ & 1 & $\mathrm{x}$ & $x$ & 1 & $x$ & & $\begin{array}{l}x \\
x\end{array}$ & $x$ & $\begin{array}{r}x \\
1\end{array}$ & & & & 1 & & $x$ \\
\hline $\begin{array}{l}\text { Nodosaria sp. } \\
\text { Nonion auriculata } \\
\text { N. barleeanum } \\
\text { Nonion sp. } \\
\text { Nonionella atlantica }\end{array}$ & & $x$ & $x$ & 4 & $x$ & $\mathrm{x}$ & 1 & 3 & $\begin{array}{l}x \\
x\end{array}$ & & $x$ & $x$ & & & & $x$ & $x$ \\
\hline $\begin{array}{l}\text { N. turigida } \\
\text { Nonionellina labradorica } \\
\text { Oolina borealis } \\
\text { O. globosa } \\
\text { O. hexagona }\end{array}$ & 3 & 4 & 4 & $\begin{array}{l}2 \\
\mathrm{x}\end{array}$ & $\mathrm{x}^{4}$ & 5 & 2 & 2 & 1 & 2 & 5 & 2 & $\mathrm{x}^{2}$ & $\mathrm{x}$ & 3 & 3 & 2 \\
\hline $\begin{array}{l}\text { Ophthalmidium acutimargo } \\
\text { Oridorsalis umbonatus } \\
\text { Patellina corrugata } \\
\text { Parafrondiculeria helenae } \\
\text { Planulina wuellerstorfi }\end{array}$ & $\mathrm{x}$ & & & & & $x$ & & & & & $\mathrm{x}$ & & & & & & \\
\hline $\begin{array}{l}\text { Psammosphaera fusca } \\
\text { Pseudopolymorphina novangliae } \\
\text { Pullenia bulloides } \\
\text { Pyrgo williamsoni } \\
\text { Quinqueloculina agglutinans }\end{array}$ & 1 & $\begin{array}{l}\mathrm{x} \\
\mathrm{x}\end{array}$ & $\begin{array}{l}\mathrm{x} \\
\mathrm{x}\end{array}$ & & & $x$ & & & & & $x$ & & $x$ & & & $x$ & $x$ \\
\hline $\begin{array}{l}\text { Q. cultrata } \\
\text { Q. seminulum } \\
\text { Q. stalkeri } \\
\text { Rectoglandulina torrida } \\
\text { Recurvoides turbinatus }\end{array}$ & & $x$ & $\begin{array}{l}x \\
x\end{array}$ & $x$ & $\begin{array}{l}\mathrm{x} \\
\mathrm{x}\end{array}$ & $\mathrm{x}$ & & 2 & 1 & 1 & & 1 & 1 & & & $x$ & $\begin{array}{l}1 \\
1\end{array}$ \\
\hline $\begin{array}{l}\text { Reophax guttifer } \\
\text { R. scottii } \\
\text { Robertinoides charlottensis } \\
\text { Rosalina columbiensis } \\
\text { Saccammina difflugiformis }\end{array}$ & $\mathrm{X}$ & $x$ & & $\begin{array}{l}x \\
1\end{array}$ & & & & & & & $\mathrm{X}$ & & $x$ & & & & \\
\hline $\begin{array}{l}\text { Sphaeroidina bulloides } \\
\text { Spiroplectammina biformis } \\
\text { Stetsonia horvathi } \\
\text { Stilostomella bradyi } \\
\text { Textularia conica }\end{array}$ & 2 & 1 & 1 & $\underset{2}{x}$ & & & & & & $\mathrm{x}$ & $\begin{array}{l}\mathrm{x} \\
\mathrm{x}\end{array}$ & & & & & & \\
\hline $\begin{array}{l}\text { T. earlandi } \\
\text { T. torquata } \\
\text { Tosaia hanzawai } \\
\text { Trifarina fluens } \\
\text { Triloculina arctica }\end{array}$ & & $\begin{array}{l}x \\
x \\
x\end{array}$ & $\begin{array}{l}\mathrm{x} \\
\mathrm{x}\end{array}$ & & & $x$ & & 2 & 1 & & $\begin{array}{l}\mathrm{x} \\
\mathrm{x}\end{array}$ & $\begin{array}{l}1 \\
1 \\
1\end{array}$ & 1 & $x$ & & & $\mathrm{x}$ \\
\hline $\begin{array}{l}\text { T. carinata } \\
\text { Trochammina bullata } \\
\text { T. "inflata" } \\
\text { T. lobata } \\
\text { T. nitida }\end{array}$ & 5 & 3 & 1 & $x$ & $\mathrm{x}$ & & & & $\mathrm{x}$ & & 1 & & $\mathrm{x}$ & & & $\mathrm{x}$ & $\begin{array}{l}1 \\
x\end{array}$ \\
\hline $\begin{array}{l}\text { T. ochracea } \\
\text { T. squamata } \\
\text { Uvigerina asperula } \\
\text { U. peregrina } \\
\text { Valvulinera laevigata }\end{array}$ & 25 & $\begin{array}{l}21 \\
X\end{array}$ & $\begin{array}{l}8 \\
1\end{array}$ & & & & & & 1 & & $\begin{array}{r}13 \\
1\end{array}$ & & & $x$ & & & $x$ \\
\hline
\end{tabular}

and some of our own work on the Nova Scotia slope (Williamson et al., 1984; Schroeder, 1986) shows that Elphidium excavatum can be a dominating species at slope depths, but not with $C$. reniforme as a subdominant. Although there is little doubt that the ElphidiumCassidulina fauna represents glacial bottom-water conditions, an interglacial planktonic foraminiferal fauna has been noted from these "glacial" samples at Site 612
(Poag and Low, this volume), so that the question remains open whether the Elphidium-Cassidulina fauna is in place.

The $E$. takayanagii fauna appears to have no modern or fossil correspondent. Leslie (1965) reports finding $E$. takayanagii in Hudson's Bay, but usually in abundances of less than $10 \%$. Schafer and Cole (1978) also report the species in Chaleur Bay (New Brunswick) in cold, sa- 
Table 1 (continued).

\begin{tabular}{|c|c|c|c|c|c|c|c|c|c|c|c|c|c|c|c|c|c|c|c|}
\hline 8.13 & 9.13 & 9.57 & 10.13 & 10.63 & 11.07 & 11.63 & 12.13 & 14.75 & 15.03 & 15.73 & 16.25 & 16.73 & 17.23 & 17.75 & 18.27 & 18.73 & 19.37 & 19.93 & 20.73 \\
\hline $2-3$ & $2-3$ & $2-4$ & $2-4$ & $2-4$ & $2-5$ & $2-5$ & $2-5$ & $3-1$ & $3-1$ & $3-1$ & $3-2$ & $3-2$ & 3.2 & 3.3 & $3-3$ & $3-3$ & $3-4$ & $3-4$ & $3-5$ \\
\hline 24 & 24 & 27 & 15 & 30 & 25 & 26 & 32 & 23 & 21 & 35 & 28 & 19 & 26 & 21 & 26 & 28 & 21 & 17 & 26 \\
\hline 1112 & 265 & 836 & 1100 & 305 & 493 & 574 & 481 & 944 & 276 & 461 & 412 & 780 & 185 & 1016 & 1580 & 2040 & 2648 & 1488 & 5392 \\
\hline 8 & 6 & 11 & 6 & 2 & 4 & 6 & 9 & 6 & 9 & 5 & 5 & 11 & 5 & 14 & 4 & 3 & 4 & 4 & 5 \\
\hline$x$ & & 1 & & & $x$ & $x$ & & & & $x$ & $x$ & $x$ & $x$ & & $x$ & $x$ & & & \\
\hline$x$ & & $x$ & 1 & $x$ & & $x$ & $x$ & $x$ & & $\begin{array}{l}x \\
x \\
\end{array}$ & 1 & & $\begin{array}{l}x \\
2 \\
\end{array}$ & $x$ & & $\begin{array}{r}X \\
2\end{array}$ & & & $\begin{array}{l}1 \\
1 \\
\end{array}$ \\
\hline$x$ & $x$ & $\begin{array}{l}1 \\
x \\
x\end{array}$ & & $\begin{array}{l}1 \\
1\end{array}$ & $\mathbf{x}^{1}$ & $x$ & $\mathrm{x}$ & 1 & 5 & 2 & $x^{1}$ & & 1 & 1 & $\mathrm{x}^{2}$ & $\underset{1}{x}$ & $\begin{array}{l}2 \\
1\end{array}$ & $\mathrm{x}^{2}$ & $x$ \\
\hline & & $x$ & 1 & & & & & & & $x$ & & & & & & & & & \\
\hline$x$ & & $x$ & & & & & & & $x$ & & 1 & 2 & & & $x$ & & $x$ & & $x$ \\
\hline$x$ & $\begin{array}{l}x \\
1\end{array}$ & 1 & 1 & $\begin{array}{l}1 \\
1\end{array}$ & $x$ & 2 & $\begin{array}{l}x \\
x\end{array}$ & & 4 & $\begin{array}{r}3 \\
\times \\
\end{array}$ & 2 & 2 & $x$ & $x$ & 1 & $x$ & 1 & 1 & 1 \\
\hline & & & & & & $x$ & & $x$ & $x$ & & & & & $x$ & & & & & \\
\hline & & & & 1 & & & & $x$ & 1 & $\underset{2}{x}$ & 1 & 1 & 2 & & & & & & $x$ \\
\hline$x$ & $\begin{array}{l}x \\
1\end{array}$ & & & $\begin{array}{l}x \\
2\end{array}$ & $x$ & $\underset{\mathrm{x}}{\mathrm{x}}$ & $x$ & $x^{1}$ & 1 & $x$ & $\begin{array}{l}x \\
x\end{array}$ & 3 & $\begin{array}{c}x \\
1\end{array}$ & & & $x$ & $\begin{array}{l}x \\
X\end{array}$ & $x$ & \\
\hline & $\begin{array}{l}\mathrm{x} \\
\mathrm{x} \\
\mathrm{x}\end{array}$ & $\underset{1}{x}$ & $x$ & $\begin{array}{l}1 \\
1\end{array}$ & 1 & & $\underset{1}{x}$ & 1 & $\begin{array}{l}1 \\
1\end{array}$ & $\begin{array}{l}\mathrm{X} \\
\mathrm{X} \\
2\end{array}$ & & & . & & & & & & \\
\hline & $\begin{array}{l}1 \\
x\end{array}$ & & & 1 & $\begin{array}{l}x \\
x\end{array}$ & $x$ & $\begin{array}{l}x \\
x\end{array}$ & $x$ & & $\begin{array}{l}x \\
x\end{array}$ & $x$ & & & & & $x$ & $x$ & & $\begin{array}{l}x \\
x\end{array}$ \\
\hline
\end{tabular}

line water of the Gaspé Current. Both of these reported occurrences are in shallow (less than $100 \mathrm{~m}$ ) water. It is quite clear that, at least in the upper Pleistocene (0.5$25 \mathrm{~m}$ at Site 613), this fauna characterized the glacial bottom water at $2400 \mathrm{~m}$, as opposed to the ElphidiumCassidulina fauna at $1400 \mathrm{~m}$. It appears that bottomwater conditions were less affected by glacial conditions at $2400 \mathrm{~m}$ than at $1400 \mathrm{~m}$, but the E. takayanagii com- ponent certainly does not denote typical interglacial conditions as we know them today. The $E$. takayanagii fauna may be the high-salinity, cold-water counterpart of the slightly lower salinity, more tolerant $E$. excavatumC. reniforme fauna.

Phleger (1942) studied a series of short cores (1-3 m) from this region, taken in water depths of from 500 to $5000 \mathrm{~m}$. In many of his cores, he observed the same 
Table 1 (continued).

\begin{tabular}{|c|c|c|c|c|c|c|c|c|c|c|c|c|c|c|c|c|}
\hline Depth of core $(m)$ & 21.23 & 21.73 & 24.03 & 24.83 & 25.33 & 25.87 & 26.83 & 27.37 & 28.33 & 28.83 & 29.33 & 29.83 & 30.35 & 30.83 & 31.33 & 31.83 \\
\hline Core-Section & $3-5$ & $3-5$ & $4-1$ & $4-1$ & $4-1$ & $4-2$ & $4-2$ & $4-3$ & $4-3$ & $4-4$ & $4-4$ & 4.4 & $4-5$ & $4-5$ & $4-5$ & $4-6$ \\
\hline No. of species & 19 & 22 & 22 & 15 & 19 & 23 & 29 & 18 & 13 & 24 & 21 & 23 & 25 & 14 & 24 & 22 \\
\hline $\begin{array}{l}\text { Total number of } \\
\text { individuals } / 10 \mathrm{~cm}^{3}\end{array}$ & 1992 & 1092 & 3408 & 628 & 2148 & 3816 & 890 & 176 & 472 & 557 & 548 & 662 & 5760 & 2992 & 1908 & 1136 \\
\hline $\begin{array}{l}\text { Hoeglundina elegans } \\
\text { Hyperammina sp. } \\
\text { Islandiella teretis } \\
\end{array}$ & 9 & 4 & 11 & 15 & 3 & 5 & 5 & 16 & 11 & 23 & 26 & 31 & 14 & 7 & 8 & 4 \\
\hline $\begin{array}{l}\text { Karreriella bradyi } \\
\text { Lagena spp. } \\
\text { Lenticulina denticulifera } \\
\text { Lenticulina spp. } \\
\text { Miliolinella subrotunda } \\
\end{array}$ & & 1 & 1 & $\mathrm{x}$ & $\begin{array}{l}\mathrm{x} \\
\mathrm{x}\end{array}$ & $\mathrm{x}$ & $\mathrm{x}$ & & & & & & $\mathrm{x}$ & 1 & $\begin{array}{l}\mathrm{x} \\
\mathrm{x}\end{array}$ & $\mathrm{x}$ \\
\hline $\begin{array}{l}\text { Nodosaria sp. } \\
\text { Nonion auriculata } \\
\text { N. barleeanum } \\
\text { Nonion sp. } \\
\text { Nonionella atlantica } \\
\end{array}$ & & & $\begin{array}{l}1 \\
1\end{array}$ & 1 & 3 & $\begin{array}{l}x \\
1 \\
\end{array}$ & 1 & $\mathrm{x}$ & $\begin{array}{c}x \\
1\end{array}$ & 2 & 5 & 4 & $\begin{array}{r}6 \\
12 \\
\end{array}$ & $\begin{array}{r}4 \\
\times \\
\end{array}$ & $\begin{array}{c}x \\
1 \\
\end{array}$ & $\begin{array}{l}4 \\
2 \\
\end{array}$ \\
\hline $\begin{array}{l}\text { N. turigida } \\
\text { Nonionellina labradorica } \\
\text { Oolina borealis } \\
\text { O. globosa } \\
\text { O. hexagona } \\
\end{array}$ & $\begin{array}{l}1 \\
2\end{array}$ & $\begin{array}{l}2 \\
1\end{array}$ & 1 & 2 & $x$ & $\begin{array}{l}1 \\
1\end{array}$ & $\begin{array}{l}1 \\
1\end{array}$ & $\begin{array}{l}3 \\
2\end{array}$ & & $x$ & 2 & $\begin{array}{l}2 \\
1\end{array}$ & $x$ & 1 & $\mathrm{x}^{4}$ & 8 \\
\hline $\begin{array}{l}\text { Ophthalmidium acutimargo } \\
\text { Oridorsalis umbonatus } \\
\text { Patellina corrugata } \\
\text { Parafrondiculeria helenae } \\
\text { Planulina wuellerstorfi }\end{array}$ & & & & & & & & & & & & & $\mathrm{x}$ & & & \\
\hline $\begin{array}{l}\text { Psammosphaera fusca } \\
\text { Pseudopolymorphina novangliae } \\
\text { Pullenia bulloides } \\
\text { Pyrgo williamsoni } \\
\text { Quinqueloculina agglutinans }\end{array}$ & & & $x$ & & & $\begin{array}{l}\mathrm{x} \\
\mathrm{x}\end{array}$ & $\begin{array}{l}x \\
x \\
1\end{array}$ & $x$ & & $\begin{array}{l}x \\
x\end{array}$ & & $\begin{array}{l}x \\
x\end{array}$ & & & $\mathrm{x}$ & \\
\hline $\begin{array}{l}\text { Q. cultrata } \\
\text { Q. seminulum } \\
\text { Q. stalkeri } \\
\text { Rectoglandulina torrida } \\
\text { Recurvoides turbinatus }\end{array}$ & 1 & 1 & 1 & & & $\mathrm{x}$ & 9 & 3 & 2 & 2 & 5 & 5 & $\mathrm{x}$ & & 2 & 7 \\
\hline $\begin{array}{l}\text { Reophax guttifer } \\
\text { R. scottii } \\
\text { Robertinoides charlottensis } \\
\text { Rosalina columbiensis } \\
\text { Saccammina difflugiformis }\end{array}$ & & $\mathrm{x}$ & & & & & & & & & & & & & & \\
\hline $\begin{array}{l}\text { Sphaeroidina bulloides } \\
\text { Spiroplectammina biformis } \\
\text { Stetsonia horvathi } \\
\text { Stilostomella bradyi } \\
\text { Textularia conica }\end{array}$ & $\mathrm{x}$ & $x$ & & & & $x$ & 3 & & 1 & & 1 & 1 & $x$ & & 11 & 1 \\
\hline $\begin{array}{l}\text { T. earlandi } \\
\text { T. torquata } \\
\text { Tosaia hanzawai } \\
\text { Trifarina fluens } \\
\text { Triloculina arctica }\end{array}$ & $x$ & & & & & & $x$ & & & & $x$ & & & & & \\
\hline $\begin{array}{l}\text { T. carinata } \\
\text { Trochammina bullata } \\
\text { T. "inflata" } \\
\text { T. lobata } \\
\text { T. nitida } \\
\end{array}$ & & & $x$ & & & & & $x$ & & & & & & & & $x$ \\
\hline $\begin{array}{l}\text { T. ochracea } \\
\text { T. squamata } \\
\text { Uvigerina asperula } \\
\text { U. peregrina } \\
\text { Valvulinera laevigata }\end{array}$ & & $x$ & & & & $\mathrm{x}$ & $\mathrm{x}$ & & & $x$ & & & & & $x$ & \\
\hline
\end{tabular}

rapid transition from a Uvigerina fauna to an Elphidium fauna in the upper $1 \mathrm{~m}$. But he did not report subsurface faunas resembling the E. takayanagii fauna. His work does show, though, that the Holocene section is thin over a wide region, not just at our two Leg 95 sites.

In the lower Pleistocene sections of Sites 612 and 613, where we cannot compare between sites, there are some significant events as well. At Site 612, Epistominella exigua becomes common below $15 \mathrm{~m}$, and this coincides with a general increase in total populations from 500 to over $2000 / 10 \mathrm{~cm}^{3}$. E. exigua has been shown to be an indicator of North Atlantic Deep Water (NADW) and the Western Boundary Undercurrent (WBU) (e.g., Schnitker, 1979; Schafer and Cole, 1982). The WBU presently 
Table 1 (continued).

\begin{tabular}{|c|c|c|c|c|c|c|c|c|c|c|c|c|c|c|c|c|c|c|}
\hline 32.33 & 32.83 & 33.63 & 34.10 & 34.60 & 34.93 & 35.37 & 35.93 & 36.43 & 37.41 & 37.93 & 38.37 & 39.43 & 41.93 & 43.43 & 45.93 & 53.43 & 54.93 & 56.43 \\
\hline $4-6$ & $4-6$ & $5-1$ & $5-1$ & $5-1$ & $5-1$ & $5-2$ & $5-2$ & $5-2$ & $5-3$ & $5-3$ & $5-4$ & $5-4$ & $6-1$ & $6-2$ & $7-1$ & 8-1 & $8-2$ & $8-3$ \\
\hline 22 & 17 & 26 & 27 & 23 & 31 & 20 & 21 & 18 & 27 & 25 & 25 & 29 & 34 & 9 & 31 & 27 & 38 & 7 \\
\hline 1580 & 4424 & 1424 & 1508 & 936 & 890 & 812 & 1068 & 496 & 480 & 473 & 452 & 672 & 2162 & 16 & 129 & 2208 & 1776 & 9 \\
\hline \multirow[t]{2}{*}{18} & 1 & 6 & 22 & 12 & 5 & 7 & 15 & 3 & 1 & & & 4 & $x$ & & 2 & $\begin{array}{l}2 \\
1\end{array}$ & & \\
\hline & $x$ & $x$ & $x$ & & & & & & & 1 & & $x$ & & 6 & 2 & 1 & $\stackrel{1}{x}$ & \\
\hline 11 & $x$ & $\begin{array}{l}4 \\
1\end{array}$ & $x$ & & $x$ & & & $\begin{array}{r}1 \\
14\end{array}$ & $\mathrm{X}$ & & 1 & & $\begin{array}{r}2 \\
x\end{array}$ & & 1 & 1 & 6 & 11 \\
\hline \multirow[t]{2}{*}{$\begin{array}{l}5 \\
2\end{array}$} & & 3 & 1 & 2 & $\begin{array}{l}\mathrm{l}_{\mathrm{x}} \\
\mathrm{x}\end{array}$ & $\begin{array}{l}1 \\
x \\
x\end{array}$ & $\begin{array}{l}4 \\
1\end{array}$ & & 2 & $\begin{array}{l}1 \\
1\end{array}$ & $\begin{array}{l}x \\
x\end{array}$ & 2 & $\begin{array}{r}2 \\
x\end{array}$ & 6 & 1 & $x$ & $\begin{array}{l}x \\
x \\
x\end{array}$ & 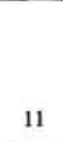 \\
\hline & & & & & & & & & & $x$ & & & $x$ & & 1 & $\mathrm{x}$ & 1 & \\
\hline $\begin{array}{l}X \\
X\end{array}$ & & 2 & & $\begin{array}{l}\mathrm{x} \\
\mathrm{x}\end{array}$ & $\begin{array}{l}\mathrm{X} \\
\mathrm{x}\end{array}$ & & & & $x$ & & & & $x$ & & 1 & & $\begin{array}{r}x \\
3 \\
x\end{array}$ & \\
\hline \multirow[t]{2}{*}{1} & 5 & 1 & 2 & 1 & $\begin{array}{l}x \\
X\end{array}$ & 9 & 5 & & $\begin{array}{l}x \\
2\end{array}$ & & 5 & 2 & & & & $\begin{array}{l}x \\
x\end{array}$ & & \\
\hline & $x$ & & & & & & & & & & & & & & & & & \\
\hline \multirow[t]{4}{*}{$x$} & & $\mathrm{x}$ & & & & & & & 5 & $\begin{array}{l}8 \\
\mathrm{x}\end{array}$ & 29 & $\mathrm{x}$ & $\begin{array}{l}x \\
x \\
x\end{array}$ & & 6 & 6 & 14 & \\
\hline & & & & $x$ & $\begin{array}{l}X \\
X\end{array}$ & $x$ & $x$ & & & & & 1 & & & & & & \\
\hline & & & & & & & & & & & & & & & 1 & & $x$ & \\
\hline & $\mathrm{x}$ & & $\mathrm{x}$ & $\mathrm{x}$ & & & & 19 & $\begin{array}{r}47 \\
4 \\
\times\end{array}$ & 60 & $\begin{array}{r}34 \\
5 \\
1\end{array}$ & $\mathrm{x}$ & $\begin{array}{l}11 \\
\mathrm{X}\end{array}$ & 6 & $\begin{array}{l}1 \\
1\end{array}$ & $\begin{array}{r}51 \\
5 \\
\times\end{array}$ & $\begin{array}{r}2 \\
11 \\
x\end{array}$ & \\
\hline
\end{tabular}

occupies a depth range below $2500 \mathrm{~m}$, well below the water depth at Site 612. Ledbetter and Balsam (1985) report that the axis of the WBU has varied by as much as $500 \mathrm{~m}$ in the last $25 \mathrm{kyr}$. but has not been above about $3500 \mathrm{~m}$. If $E$. exigua does indicate the presence of the WBU, then the WBU was significantly shallower in previous interglacial cycles. It is not likely that the WBU would have been present at Site 612 and absent at Site 613 in the same time interval. Hence, the absence of any $E$. exigua fauna in Site 613 samples indicates that the time-equivalent interval of the 15 to $35 \mathrm{~m}$ section at Site 612 was not sampled or was missing at Site 613 . There is overlap at these hole depths between the two sites (we have $19-29 \mathrm{~m}$ in Hole 613 ), so obviously sedimentation 
D. B. SCOTT

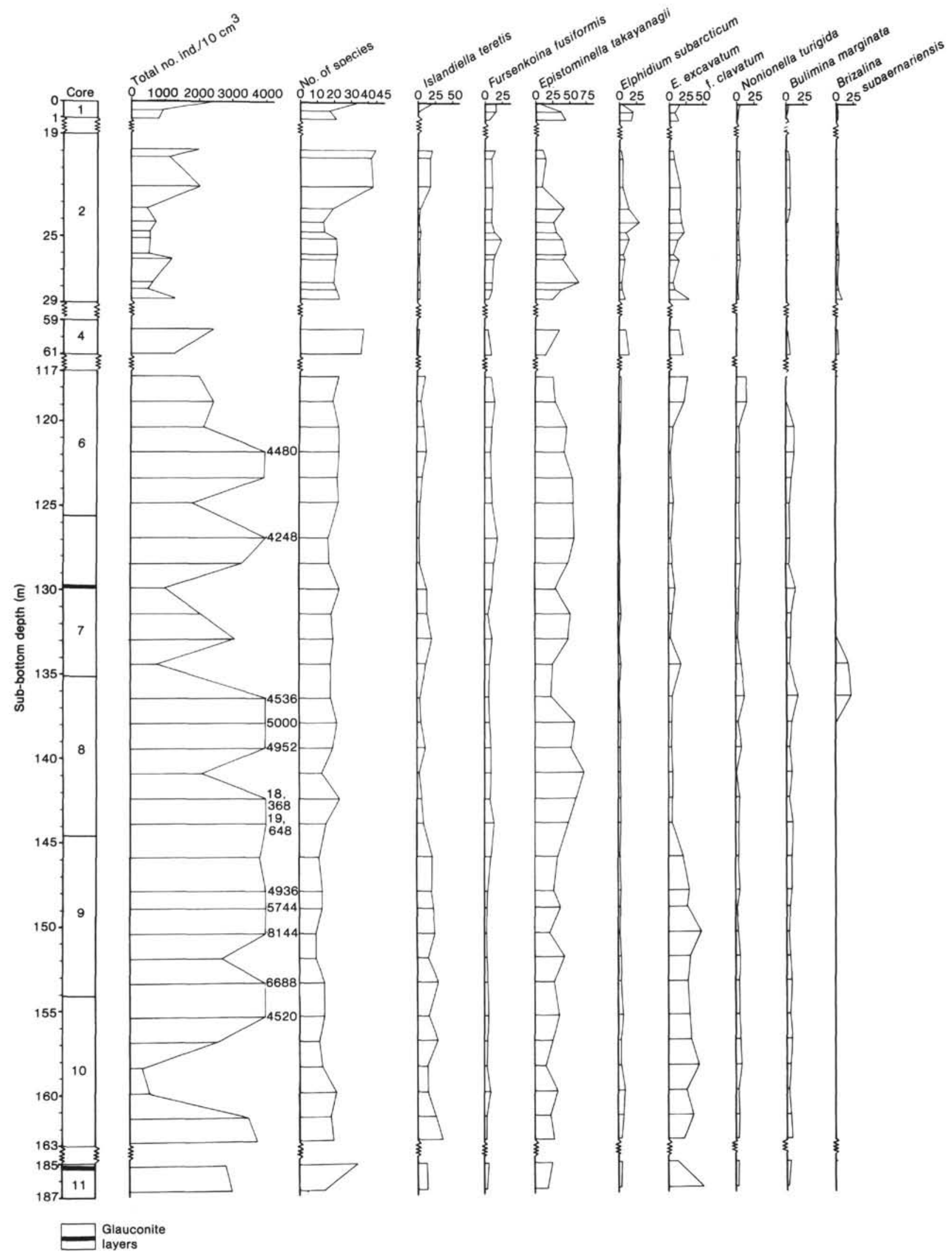

Figure 2. Percentage occurrences of selected species, Hole 613. Format same as in Figure 1. 
rates were quite different between sites, and/or there are hiatuses (seismics at Site 613 show several hiatuses in the Pleistocene).

It is also of note that the surface fauna (i.e., presentday interglacial) does not recur downcore at Site 612 but does (19-22 m) at Site 613. In comparing the two sequences, it appears that major sections are missing from both sites, and without some absolute dating controls it will be very difficult to determine exactly how to correlate the Pleistocene records from the two sites.

The only characteristic common to both sites appears at the bases of both sections, where Elphidium faunas do occur with glauconite layers and reworked TertiaryCretaceous microfossils. But the basal faunas differ in many aspects (no Uvigerina at Site 613, low Islandiella teretis at Site 612, etc.), and it is unclear whether these sections are correlatable.

\section{ACKNOWLEDGMENTS}

Drs. K. Miller and W. Poag provided the author with many useful comments and useful pieces of information, and reviewed the fina manuscript. B. Deonarine (Atlantic Geoscience Centre) kindly took all the SEM photos of the foraminifers. Part of laboratory preparations were done by C. Younger, V. Baki, K. Mackinnon, and L. Dobbin (Dalhousie). Funding was supplied by Natural Sciences and Engineering Research Council (Canada) Strategic and Operating grants to Scott.

\section{REFERENCES}

Asano, K., 1938. On the Japanese species of Bolivina and its allied genera. J. Geol. Soc. Japan, 45:600-609.

Bailey, J. W., 1851. Microscopical examination of soundings made by the United States Coast Survey of the Atlantic coast of the United States. Smithson. Contr. Knowl., 2(3):1-15.

Barker, R. W., 1960. Taxonomic notes on the species figured by H. B. Brady in his report on the Foraminifera dredged by H.M.S. Challenger during the years 1873-76. Soc. Econ. Paleontol. Mineral., Spec. Publ., 9.

Bell, D. L., and Goodell, H. G., 1967. A comparative study of glauconite and the associated clay fraction in modern marine sediments. Sedimentology, 9:169-202.

Brady, H. B., 1878. On the reticularian and radiolarian rhizopoda (Foraminifera and Polycystina of the North-Polar Expedition of 1875-76. Ann. Mag. Nat. Hist., Ser. 5, 1:425-440.

1879. Notes on some of the reticularian Rhizopoda of the Challenger Expedition: Part 1, on new or little known arenaceous types. Q. J. Microsc. Sci., new ser., 19:20-63.

1881. On some Arctic Foraminifera from soundings obtained on the Austro-Hungarian North Polar Expedition of 1872-76. Ann. Mag. Nat. Hist., 8:393-418.

1884. Report on the Foraminifera dredged by the H.M.S Challenger during the years 1873-1876. Reports of scientific results from Explorer. Voyage of the H.M.S. Challenger, Zoology, 9: $1-814$, pl. 1-115.

Chapman, F., 1941. Report on foraminiferal soundings and dredgings of the F.I.S. "Endeavour" along the continental shelf of the southeast coast of Australia. Trans. R. Soc. S. Austral., 65(2):145-211.

Chaster, G. W., 1892. Foraminifera. First Report of the Southport Society of Natural Science, 1890-91: pp. 54-72.

Cole, F. E., 1981. Taxonomic notes on the bathyal zone benthonic foraminifera species off Northeast Newfoundland. Bedford Inst. Rept. Ser., B1-R-81-7, Dartmath, N.S., 120 pp. 20 pls.

Cronin, T. M., 1979. Foraminifera and ostracode species diversity in the Pleistocene Champlain Sea of the St. Lawrence Lowlands. J. Paleontol, 53:233-244.

Cushman, J. A., 1911. A monograph of the Foraminifera of the North Pacific Ocean, Pt. 2, Textularidae. U.S. Nat. Mus. Bull., 71:1-108. 1913. A monograph of the Foraminifera of the North Pacific Ocean, Pt. 4, Lagenidae. U.S. Nat. Mus. Bull., 74.

1918-1931. The Foraminifera of the Atlantic Ocean. U.S. Nat. Mus. Bull., 104 (Pts. 1-8). 1922a. The Foraminifera of the Atlantic Ocean, Pt. 3, Textulariidae. U.S. Natl. Mus. Bull., 104(3):1-149.

1922b. Results of the Hudson Bay expedition, 1920: I. The Foraminifera. Can. Biol. Board, Contr. Can. Biol. (1921), Toronto (1922), 9:135-147.

1922c. The Byram Calcareous Marl of Mississippi and its Foraminifera. U.S. Geol. Surv. Prof. Pap., 129-E, 87-122.

1923. Foraminifera of the Atlantic Ocean, Pt. 4, Lagenidae. U.S. Natl. Mus. Bull., 104(4):1-228.

1925. Recent Foraminifera of British Columbia. Contrib. Cush. Lab. Foram. Res., 1(2):38-47.

1927. An outline of a reclassification of the Foraminifera. Cushman Lab. Foram. Res. Contr., 3:1-105.

1933. Foraminifera: Their classification and economic use. Cushman Lab. Foram. Res. Contr., Spec. Publ., 4.

1944. Foraminifera from the shallow water of the New England coast. Cushman Lab. Foram. Res., Spec. Publ., 12:1-37. 1947. New species and varieties of Foraminifera from off the southeast coast of the United States. Cushman Lab. Foram. Res. Contr., 23:86-92.

Cushman, J. A., and Todd, R., 1947. A foraminiferal fauna from Amchitka Island, Alaska. Cushman Lab. Foram. Res. Contr., 23:6072 .

Czjzek, J., 1848. Beitrag zur Kenntniss der fossilen Foraminiferen des Wiener Beckens. Haidingers Naturwiss. Abh., 2(1):137-150.

Dawson, G. M., 1870. On Foraminifera from the Gulf and River St. Lawrence. Can. Nat. Q. J. Sci., 5:172-180.

Dawson, J. W., 1860. Notice of Tertiary fossils from Labrador, Maine, etc., and remarks on the climate of Canada in the newer Pliocene or Pleistocene period. Can. Nat., 5:188-200.

d'Orbigny, A. D., 1826. Tableau méthodique de la classes des céphalopods. Ann. Sci. Nat. Paris, Ser. 1, 7:245-314.

1839. Voyage dans l'Amérique Méridionale-Foraminiferes, (Vol. 5, Pt. 8): Paris (Pitois-Levrault et Cie); Strasbourg (V. Levrault).

1846. Foraminifères fossiles du Bassin Tertiare de Vienne (Autriche). Paris (Gide et Cie).

Drake, C. L., Ewing, M., and Sutton, G. H., 1959. Continental margins and geosynclines: The east coast of North America north of Cape Hatteras. Physics and Chemistry of the North (Vol. 3): New York (Pergamon Press), 110-198.

Erland, A., 1934. Foraminifera, Part III. The Falklands sector of the Antarctic (excluding south Georgia). Discovery Repts., 10:1-208.

Feyling-Hanssen, R. W., 1972. The Pleistocene/Holocene boundary in marine deposits from the Oslo Fjord area. Boreas., 1:241-246.

Fichtel, L., and Moll, J. P., 1798. Testacea microscopica, aliaque minuta ex generibus Argonauta et Nautilus, ad naturam picta et descripta (Microscopische und andere klein Schalthiere aus den Geschechter Argonaute und Schiffer): Wien (Camesina), reprinted in 1803.

Gervirtz, J. L., Park, R. A., and Friedman, G. M., 1971. Paraecology of benthonic foraminifera and associated micro-organisms of the continental shelf off Long Island, New York. J. Paleontol., 45: 153-177.

Green, K. E., 1960. Ecology of some Arctic Foraminifera. Micropaleontology, 6:57-78.

Heron-Allen, E., and Earland, A., 1930. The Foraminifera of the Plymouth District, II. J. R. Micr. Soc., London, Ser. 3, 50:161-199.

Hoeglund, H., 1947. Foraminifera in the Gullmar Fjord and Skagerak. Zool. Bidrag., fran., Uppsala Bd. 26.

Iwasa, S., 1955. Biostratigraphy of the Iswasagawa Group in Honjo and its environ, Akita Prefecture. J. Geol. Soc. Tokyo, 61:1-18.

Kafescioglu, I. A., 1975. Quantitative distribution of foraminifera on the continental shelf and uppermost slope off Massachusetts. Micropaleontology, 21:261-305.

Kanmacher, F., 1798. Adams Essays on the Microscope: The Second Edition, with Considerable Additions and Improvements: London (Dillon and Keating).

Ledbetter, M. T., and Balsam, W. L., 1985. Paleoceanography of the Deep Western Boundary Undercurrent on the North American continental margin for the past 25,000 yr. Geology, 13:181-184.

Leslie, R. J., 1965. Ecology and paleoecology of Hudson Bay foraminifera. Bedford Inst. Oceanog. Rept., $65-6$ (unpubl. manuscript).

Linné, C., 1758. Systema naturae per regna tria naturae, secundum classes, ordines, genera, species, cum characteribus, differentiis, 
synonymis, locis (Vol. 1) (10th ed.): Lipsiae (G. Engelmann), 1824.

Loeblich, A. R., Jr., and Tappan, H., 1953. Studies of Arctic Foraminifera. Smithson. Misc. Collect., 121.

1954. New names for two foraminiferal homonyms. Wash. Acad. Sci. J., 44(12):384 only.

1964. Sarcodina, chiefly "Thecamoebians" and Foraminiferida. In Moore, R. C. (Ed.), Treatise on Invertebrate Paleontology (Pt. C. Vols. 1 and 2): Lawrence (Kansas Univ. Press).

McRae, S. G., 1972. Glauconite. Earth Sci. Rev., 8:397-440.

Miller, A. A. L., Scott, D. B., and Medioli, F. S., 1982. Elphidium excavatum (Terquem): Ecophenotypic versus subspecific variation. $J$. Foram. Res., 12:116-144.

Miller, K. G., and Lohmann, G. P., 1982. Environmental distribution of Recent benthic foraminifera on the northeast United States continental slope. Geol. Soc. Am. Bull., 93:200-206.

Montagu, G., 1803. Testacea Britannica, or Natural History of British Shells, Marine, Land, and Fresh-water, Including the Most Minute: Romsey, England, (J. S. Hollis).

Moulotte and Blanc-Vernet, in press. Quaternary benthonic foraminifera from DSDP Leg 93. In van Hinte, J., Wise, S. W., Jr., et al., Init. Repts. DSDP, 93: Washington (U.S. Govt. Printing Office).

Murray, J. W., 1969. Recent foraminifera from the Atlantic continental shelf of the United States. Micropaleontology, 15:401-419.

Norvang, A., 1945. The zoology of Iceland, Foraminifera (2 pts., no. 2): Copenhagen and Reykjavik (Ejnar Munksgaard).

Parker, F. L., 1948. Foraminifera of the continental shelf from the gulf of Maine to Maryland. Bull. Mus. Comp. Zool. (Harvard), 100: 213-291.

1952a. Foraminiferal distribution in the Long Island-Buzzards Bay area. Bull. Mus. Comp. Zool. (Harvard), 106(10):438473.

1952b. Foraminifera species off Portsmouth, New Hampshire. Bull. Mus. Comp. Zool. (Harvard) 106(9):391-423.

Parker, W. K., and Jones, T. R., 1865. On some Foraminifera from the North Atlantic and Arctic oceans, including Davis Strait and Baffin's Bay. Phil. Trans., R. Soc. London, 155:325-441.

Parr, W. J., 1950. Foraminifera. B.A.N.Z. Antarctic Res. Exped. 192931 , Rept. Ser. B, 5(6):232-392.

Phleger, F. B., 1942. Foraminifera of submarine cores from the continental slope, Pt. 2. Bull. Geol. Soc. Am., 53:1073-1098.

Phleger, F. G., and Parker, F. L., 1951. Ecology of Foraminifera, northwest Gulf of Mexico, Pt. II. Foraminifera species. Mem. Geol. Soc. Am., 46:64.

Poag, C. W., Knebel, H. J., and Todd, R., 1980. Distribution of modern benthic foraminifers on the New Jersey outer continental shelf. Mar. Micropaleontol., 5:43-69.

Reuss, A. E., 1850. Neues Foraminiferen aus den Schichten des oesterreichischen Tertiarbeckens. K. Akad. Wiss. Wien, Math-Naturwiss. Cl., Denkschr., 1:365-390.

1851. Ueber die fossilen Foraminiferen und Entomostraceen der Septarienthere der Umgegend von Berlin. Z. Deut. Geol. Ges., 3:49-91.

Schaefer, C. T., and Cole, F. E., 1978. Distribution of foraminifera in Chaleur Bay, Gulf of St. Lawrence. Geol. Surv. Pap. (Geol. Surv. Can.) $77-30$.

1982. Living benthic foraminifera distributions on the continental slope and rise east of Newfoundland, Canada. Geol. Soc. Am. Bull., 93:207-217.

Schnitker, D., 1974. West Atlantic abyssal circulation during the past 120,000 years. Nature, 248:385-387.

1979. The deep waters of the western North Atlantic during the past 24,000 years, and re-initiation of the Western Boundary Undercurrent. Mar. Micropaleontol., 4:265-280.

Schroeder, C. J., 1986. Deep-water arenaceous foraminifera in the Northwest Atlantic Ocean [Ph.D. dissert.]. Dalhousie University, Halifax, Nova Scotia.

Schulze, F. E., 1875. Zoologische Ergebnisse der Nord-seefahrt vom 21 Juli bis 9 September, 1872. I. Rhizopoden. II. Jb. Komm. Untersuch. Deutsch. Meere in Kiel, 1872-1873: 99-114.

Schwager, C., 1866. Fossile Foraminiferen von Kar-Nicobar. NovaraExped., Geol. Theil, 2:187-268. 1877. Quadro del proposto sistema de classificazione dei foraminiferi con guscio. R. Comitata Geol. Ital. Bull., 8:18-27.
Scott, D. B., and Medioli, F. S., 1980. Post-glacial emergence curves in the Maritimes determined from marine sediments in raised basins. Proc. Can. Costal Conf. 1980, pp. 428-446.

Scott, D. B., Mudie, P. J., Vilks, G., and Younger, C. D., 1984. Latest Pleistocene-Holocene paleoceanographic trends on the continental margin of eastern Canada: Foraminiferal, Dinoflagellate, and pollen evidence. Mar. Micropaleontol., 9:181-218.

Silvestri, A., 1923. Microfauna pliocenia a Rizopodi reticuaria di copocelle presso forli. Accad. Pont., Romana Nuovi, Lincei, 76.

Streeter, S. S., 1973. Bottom water and benthonic foraminifera in the North Atlantic-glacial-interglacial contrasts. Quat. Res., 3:131141.

Takayanagi, Y. 1953. New genus and species of Foraminifera found in the Tonohama group, Kochi Prefecture, Shikoku, Japan. Inst. Geol. Paleontol. (Sendai), short pap. 5:25-36.

1960. Cretaceous Foraminifera from Hokkaido, Japan. Tohoku Univ. Sci. Rept., Ser. 2 (Geol.), 32:1-154.

Tappan, H., 1951. Northern Alaska index Foraminifera. Cushman Found. Foram. Res. Contr., 2:1-8.

Vilks, G., 1981. Late glacial-postglacial foraminifera boundary in sediments of eastern Canada, Denmark and Norway. Geosci. Can., 8: 48-55.

Vilks, G., and Rashid, M. A., 1976. Post-glacial paleoceanography of Emerald Basin, Scotian Shelf. Can. J. Earth Sci., 13:1256-1267.

Williamson, M. A., 1983. Distribution of Recent foraminifera on the Nova Scotian Shelf and Slope [Ph.D. thesis]. Dalhousie Univ., Halifax, Canada.

Williamson, M. A., Keen, C. E., and Mudie, P. J., 1984. Foraminiferal distribution on the continental margin off Nova Scotia. Mar. Micropaleontol., 9:219-239.

Williamson, W. C., 1848. On the Recent British species of the genus Lagena. Ann. Mag. Nat. Hist., Ser. 2, 1:1-20.

1858. On Recent Foraminifera of Great Britain. R. Soc. Publ., pp. 1-107.

Date of Initial Receipt: 1 May 1985

Date of Acceptance: 7 October 1985

\section{APPENDIX}

Systematic Taxonomy

Many of the most common species are illustrated in Plates 1 and 2. All species listed in Tables 1 and 2 are listed alphabetically here with their original references. Generic names are in accordance with Loeblich and Tappan (1964).

\section{Adercotryma glomerata (Brady)}

Lituola glomerata Brady, 1878, p. 433, pl. 20, figs. 1a-c.

\section{Ammobaculites agglutinans (d'Orbigny)}

Spirolina agglutinans d'Orbigny, 1846, p. 137, pl. 7, figs. 10-12.

Ammonia beccarii (Linné)

Nautilus beccarii Linné, 1758, p. 10.

\section{Ammotium cassis (Parker)}

Lituola cassis Parker, in Dawson, 1870, p. 177, 180, fig. 3.

\section{Astracolus crepidulus (Fichtel and Moll)}

Nautilus crepidula Fichtel and Moll, 1798, p. 107, pl. 19, figs. g-i.

\section{Astrononion gallowayi Loeblich and Tappan}

Astrononion gallowayi Loeblich and Tappan, 1953, p. 90, pl. 17, figs. 4-7.

\section{Bolivina decussata Brady}

Bolivina decussata Brady, 1881, p. 58

\section{Bolivina pseudoplicata Heron-Allen and Earland}

Bolivina pseudoplicata Heron-Allen and Earland, 1930, p. 81, pl. 3, figs. $36-40$. 
Bolivina subspinscens Cushman

Bolivina subspinscens Cushman, 1922a, p. 48, pl. 7, fig. 5 .

Brizalina bradyi (Asano)

(Plate 1, Fig. 8)

Bolivina bradyi Asano, 1938, p. 603.

Brizalina pseudopunctata (Hoeglund)

(Plate 1, Fig. 9)

Bolivina pseudopunctata Hoeglund, 1947, p. 273, pl. 24, fig. 5, pl. 32, figs. 23,24 .

\section{Brizalina spathulata (Williamson)}

(Plate 1, Fig. 10)

Textularia variabilis Williamson var. spathulata Williamson, 1858, p. 76 , pl. 6 , figs. 164,165 .

\section{Brizalina subaenariensis (Cushman) (Plate 1, Fig. 11)}

Bolivina subaenariensis Cushman, 1922a, p. 46, pl. 7, fig. 6.

\section{Buccella frigida (Cushman)}

Pulvinulina frigida Cushman, 1922 b, p. 144.

\section{Bulimina aculeata d'Orbigny}

(Plate 1, Fig. 3)

Bulimina aculeata d'Orbigny, 1826 , p. 269 , no. 7.

\section{Bulimina exilis Brady}

Bulimina elegans d'Orbigny var. exilis Brady, 1884, p. 399, pl. 50, figs. 5,6 .

\section{Bulimina gibba Fornasini}

Bulimina gibba Fornasini, Barker, 1960, p. 102, pl. 50, figs. 1-4.

Bulimina marginata d'Orbigny

Bulimina marginata d'Orbigny, 1826, p. 269, pl. 12, figs. 10, 12.

\section{Bulimina striata d'Orbigny}

(Plate 1, Fig. 4)

Bulimina striata d'Orbigny, Barker, 1960, p. 104, pl. 51, figs. 10, 12.

\section{Buliminella elegantissima (d'Orbigny)}

Bulimina elegantissima d'Orbigny, 1839, p. 51, pl. 7, figs. 13, 14.

\section{Cassidulina laevigata d'Orbigny} (Plate 2, Fig. 10)

Cassidulina laevigata d'Orbigny, 1826 , p. 282 , pl. 15 , figs. 4,5 .

\section{Cassidulina reniforme Norvang}

(Plate 2, Figs. 11, 12)

Cassidulina crassa d'Orbigny var. reniforme Norvang, 1945, p. 41, text figs. $6 \mathrm{c}-\mathrm{h}$.

\section{Chilostomella oolina Schwager}

Chilostomella oolina Schwager, 1877, p. 10, pl. 1, fig. 16.

\section{Cibicides lobatulus (Walker and Jacob)}

Nautilus lobatulus Walker and Jacob in Kanmacher, 1798, p. 642. pl. 14 , fig. 36 .

\section{Cibicides pseudoungerianus (Cushman)}

Truncatulina pseudoungerianus Cushman, 1922c, p. 97, pl. 20, fig. 9 .

\section{Cibicides robertsonianus (Brady)}

Truncatulina robertsoniana Brady, 1881, p. 65.

\section{Cribrostomoides canariensis (d'Orbigny)}

Nonionina canariensis d'Orbigny, 1839, p. 128, pl. 2, fig. 33.

\section{Cyclogyra involvens (Reuss)}

Operculina involvens Reuss, 1850 , p. 370 , pl. 14, fig. 20.

\section{Dentalina hirsuta (d'Orbigny)}

Nodosaria hirsuta d'Orbigny, 1826, p. 252, no. 7.

\section{Dentalina ittae Loeblich and Tappan}

Dentalina ittae Loeblich and Tappan, 1953, p. 56, pl. 10, figs. 10-12.

Dentalina subsoluta (Cushman) (Plate 1, Fig. 14)

Nodosaria subsoluta Cushman, 1923, p. 74.

Dentalina subemaciata Parr

Dentalina subemaciata Parr, 1950 , p. 329 , pl. 12, fig. 1.

\section{Discorbis cf. nitida (Williamson)}

Rotalina nitida Williamson, 1858 , p. 54 , pl. 4 , figs. 106-108.

\section{Eggerella advena (Cushman)}

Verneuilina advena Cushman, 1921, p. 141.

\section{Elphidium excavatum (Terquem) formae (Plate 1, Fig. 19)}

For complete taxonomic review of this species and its formae we refer to Miller et al., 1982

\section{Elphidium subarcticum Cushman}

(Plate 1, Fig. 18)

Elphidium subarcticum Cushman, 1944, p. 27, pl. 3, figs. 34, 35.

\section{Eoeponides pulchella (Parker)}

Pninaella? pulchella Parker, 1952b, p. 420, pl. 6, figs. 18-20.

Eponides bradyi Earland

(Plate 2, Figs. 3, 4)

Eponides bradyi Earland, 1934, p. 187, pl. 8, figs. 36-38.

Eponides tumidulus (Brady)

Truncatulina tumidula Brady, 1884, p. 666 , pl. 95 , figs. $8 \mathrm{a}-\mathrm{d}$.

Epistominella exigua (Brady)

(Plate 2, Figs. 8, 9)

Pulvinulina exigua Brady, 1884, p. 696, pl. 103, figs. 13, 14.

\section{Epistominella umbonifera (Cushman)}

Pulvinulina umbonifera Cushman, 1933, p. 90, pl. 9, figs. 9a-c.

\section{Epistominella takayanagii Iwasa} (Plate 2, Figs. 5-7)

Epistominella takayanagii Iwasa, 1955, pp. 16, 17, text figs. 4a-c.

Fursenkoina fusiformis (Williamson)

(Plate 1, Figs. 12, 13)

Bulimina pupoides d'Orbigny var. fusiformis Williamson, 1858, p. 64, pl. 5, figs. 129, 130.

\section{Gavelinopsis lobatulus (Parr) \\ (Plate 2, Figs. 16, 17)}

Discorbis lobatulus Parr, 1950, p. 354. 
Gavelinopsis translucens (Phleger and Parker)

(Plate 2, Figs. 14, 15)

"Rotalia" translucens Phleger and Parker, 1951, p. 24, pl. 12, figs. 11a, b, $12 \mathrm{a}, \mathrm{b}$.

Glabratella wrightii (Brady)

Discorbina wrightii Brady, 1881, p. 413, pl. 21, fig. 6 .

Globobulimina auriculata (Bailey)

Bulimina auriculata Bailey, 1851, p. 12, pl. 1, figs. 25-27.

Guembelitria sp.?

(Plate 1, Fig. 17)

Gyroidina soldanii (d'Orbigny)

Rotalia soldanii d'Orbigny, 1826 , p. 278 , fig. 36.

Haynesina orbiculare (Brady)

Nonionia orbiculare Brady, 1881, p. 415 , pl. 21, fig. 5 .

Heterohelix sp.?

(Plate 1, Fig. 16)

Hoeglundina elegans (d'Orbigny)

Rotalia elegans d'Orbigny, 1826, p. 276, no. 54.

Islandiella teretis (Tappan)

(Plate 2, Fig. 13)

Cassidulina teretis Tappan, 1951, p. 7, pl. 1, figs. 30a-c.

This species also includes $I$. helenae and $I$. norcrossi of other authors, and grades into Cassidulina laevigata.

\section{Karreriella bradyi (Cushman)}

Gaudryina bradyi Cushman, 1911, p. 67, fig. 107.

\section{Lenticulina denticulifera Cushman}

Lenticulina denticulifera Cushman, 1913, p. 75.

Lenticulina gibba (d'Orbigny)

Cristellaria gibba d'Orbigny, 1839, p. 63, pl. 7, figs. 20, 21.

Miliolinella subrotunda (Montagu)

Vermiculum subrotundum Montagu, 1803, p. 521.

Nonion barleeanum (Williamson)

Nonionina barleeana Williamson, 1858, p. 32, pl. 4, figs. 68, 69 .

\section{Nonion auriculata(Heron-Allen and Earland)}

Nonionella auriculata Heron-Allen and Earland, 1930, p. 192, pl. 5, figs. $68-70$.

\section{Nonionella atlantica Cushman}

Nonionella atlantica Cushman, 1947, p. 90, pl. 20, figs. 4, 5.

Nonionella turigida (Williamson)

(Plate 1, Figs. 21, 22)

Rotalina turigida Williamson, 1858, p. 50, pl. 4, figs. 95-97.

Nonionellina labradorica (Dawson)

Nonionina labradorica Dawson, 1860, p. 191, fig. 4.

Oolina borealis Loeblich and Tappan

Oolina borealis Loeblich and Tappan, 1954, p. 384, no. 12.

\section{Oolina globosa Montagu}

Oolina globosum Montagu, 1803, p. 523.
Oolina hexagona (Williamson)

Entosolenina squamosa (Montagu) var. hexagona Williamson, 1848 , p. 20, pl. 2, fig. 23.

\section{Opthalmidium acutimargo (Brady)}

Spiroloculina acutimargo Brady, 1884, p. 154, pl. 10, figs. 12-15.

Oridorsalis umbonatus (Reuss)

Rotalina umbonata Reuss, 1851, p. 75, pl. 5, figs. 35a-c.

Patellina corrugata Williamson

Patellina corrugata Williamson, 1858, p. 46, pl. 3, figs. 86-89.

\section{Parafrondicularia helenae Chapman}

Parafrondicularia helenae Chapman, 1941, pp. 154, 170, pl. 9, figs. $5 \mathrm{a}, \mathrm{b}$.

\section{Planulina wuellerstorfi (Schwager)}

Anomalina wuellerstorfi Schwager, 1866, p. 258, pl. 7, figs. 105, 107.

\section{Psammosphaera fusca Schulze}

Psammosphaera fusca Schulze, 1875, p. 113, pl. 2, fig. 8.

\section{Pseudopolymorphina novangliae (Cushman)}

Polymorphina lactea (Walker and Jacob) var. novangliae Cushman, 1923 , p. 146 , pl. 39 , figs. $6-8$.

\section{Pullenia bulloides (d'Orbigny)}

Nonionina bulloides d'Orbigny, 1826, p. 293.

Pullenia subcarinata (d'Orbigny) (Plate 1, Fig. 20)

Nonionina subcarinata d'Orbigny, 1839, p. 28, pl. 5, figs. 23, 24 . $P$. quinqueloba is a junior synonym of this species.

$$
\text { Pyrgo williamsoni (Silvestri) }
$$

Biloculina williamsoni Silvestri, 1923, p. 73.

$$
\text { Quinqueloculina agglutinans d'Orbigny }
$$

Quinqueloculina agglutinans d'Orbigny, 1839, p. 168, pl. 12, figs. 1113.

$$
\text { Quinqueloculina cultrata (Brady) }
$$

Miliolina cultrata Brady, 1881, p. 45.

$$
\text { Quinqueloculina seminulum (Linné) }
$$

Serpula seminulum Linné, 1758, p. 786.

\section{Quinqueloculina stalkeri Loeblich and Tappan}

Quinqueloculina stalkeri Loeblich and Tappan, 1953, p. 40, pl. 5, figs. 5-9.

\section{Rectoglandulina torrida (Cushman)}

Nodosaria (Glandulina) laevigata var. torrida Cushman, 1923, p. 65.

\section{Recurvoides turbinatus (Brady)}

Haplophragmium turbinatum Brady, 1881, p. 50.

$$
\text { Reophax arctica Brady }
$$

Reophax arctica Brady, 1881, p. 405, pl. 21, figs. 2a, b.

$$
\text { Reophax guttifer (Brady) }
$$

Lituola guttifer Brady, 1881, p. 49.

\section{Reophax scottii Chaster}

Reophax scottii Chaster, 1892, p. 57, pl. 1, fig. 1. 
Robertinoides charlottensis (Cushman)

Cassidulina charlottensis Cushman, 1925, p. 41, pl. 6, figs. 6, 7.

Rosalina columbiensis (Cushman)

Discorbis columbiensis Cushman, 1925, p. 43, pl. 6, fig. 13.

Saccammina difflugiformis (Brady)

Reophax difflugiformis Brady, 1879, p. 51, pl. 4, figs. 3a, b.

Sphaeroidina bulloides d'Orbigny

Sphaeroidina bulloides d'Orbigny, 1826, p. 267 , no. 1 .

\section{Spiroplectammina biformis (Parker and Jones)}

Textularia agglutinans d'Orbigny var. biformis Parker and Jones, 1865, p. 370 , pl. 15 , figs. 23,24 .

\section{Stetsonia horvathi Green}

(Plate 2, Figs. 1, 2)

Stetsonia horvathi Green, 1960, p. 72, pl. 1, figs. 6a, b.

\section{Stilostomella bradyi (Cushman)}

(Plate 1, Fig. 15)

Nodogenerina bradyi Cushman, 1927, p. 79.

\section{Textularia conica d'Orbigny}

Textularia conica d'Orbigny, 1839, p. 135, pl. 1, figs. 19, 20.

\section{Textularia earlandi Parker}

Textularia earlandi Parker, 1952a, p. 458 (footnote).

\section{Textularia torquata Parker}

Textularia torquata Parker, 1952b, p. 403, pl. 3, figs. 9-11.

\section{Tosaia hanzawai Takayanagi}

Tosaia hanzawai Takayanagi, 1953, p. 30, pl. 40, fig. 7.

\section{Trifarina fluens (Todd)}

Anglogerina fluens Todd in Cushman and Todd, 1947, p. 67, pl. 16, figs. $6,7$.

\section{Triloculina arctica (Cushman)}

Quinqueloculina arctica Cushman, 1933, p. 2, pl. 1, figs. 3a-c.

\section{Triloculina trihedra Loeblich and Tappan}

Triloculina trihedra Loeblich and Tappan, 1953, p. 45, pl. 4, fig. 10.

\section{Trochammina bullata Takayanagi}

(Plate 1, Figs. 1, 2)

Trochammina bullata Takayanagi, 1960, p. 85 , pl. 4, figs. 1a-c.

\section{Trochammina "inflata"?}

Trochammina inflata (Montagu), Williamson, 1983, p. 212, pl. 2, figs. 12,13 .

This species outwardly appears similar to Montagu's, except that $T$. inflata is a marsh species. The specimens observed here, and those of Williamson, lack the characteristic inner lining of the marsh forms. Cole (1981) also observed this form.

\section{Trochammina lobata Cushman}

Trochammina lobata Cushman, 1944, p. 18, pl. 2, fig. 10.

\section{Trochammina nitida Brady}

Trochammina nitida Brady, 1881, p. 52.

\section{Trochammina ochracea (Williamson)}

Rotalina ochracea Williamson, 1858, p. 55, pl. 4, fig. 112, pl. 5, fig. 113.

\section{Trochammina squamata Parker and Jones}

Trochammina squamata Parker and Jones, 1865, p. 407, pl. 15, figs. $30,31 \mathrm{a}-\mathrm{c}$.

\section{Uvigerina asperula Czjzek}

(Plate 1, Fig. 5)

Uvigerina asperula Czjzek, 1848, p. 146, pl. 13, figs. 14, 15.

\section{Uvigerina peregrina Cushman}

(Plate 1, figs. 6, 7)

Uvigerina peregrina Cushman, 1923, p. 166, pl. 42, figs. 7-10.

\section{Valvulinera laevigata Phleger and Parker}

Valvulinera laevigata Phleger and Parker, 1951, p. 28, pl. 13, figs. 11, 12. 
Table 2. Percentage occurrences of all benthic foraminiferal species observed in the Quaternary samples from Hole 613.

\begin{tabular}{|c|c|c|c|c|c|c|c|c|c|c|c|c|c|c|c|c|c|}
\hline Depth of core $(\mathrm{m})$ & 0.2 & 0.52 & 1.02 & 19.82 & 20.32 & 22.02 & 23.32 & 24.14 & 24.72 & 25.08 & 26.02 & 26.32 & 27.72 & 28.07 & 28.74 & 59.51 & 61.01 \\
\hline Core-Section & $1-1$ & $1-1$ & $1-1$ & $2-1$ & $2-1$ & $2-2$ & $2-3$ & $2-3$ & $2-4$ & $2-4$ & $2-5$ & $2-5$ & $2-6$ & $2-6$ & $2-6$ & $4-1$ & $4-2$ \\
\hline No. of species & 34 & 17 & 21 & 45 & 42 & 43 & 19 & 14 & 14 & 21 & 22 & 21 & 20 & 21 & 23 & 38 & 36 \\
\hline $\begin{array}{l}\text { Total number of } \\
\text { individuals } / 10 \mathrm{~cm}^{3}\end{array}$ & 2344 & 900 & 790 & 1996 & 1076 & 2120 & 466 & 696 & 556 & 532 & 518 & 1172 & 604 & 478 & 1280 & 2384 & 1300 \\
\hline $\begin{array}{l}\text { Astracolus crepidulus } \\
\text { Astrononion gallowayi } \\
\text { Bolivina decussata } \\
\text { B. pseudoplicata } \\
\text { B. subspinescens } \\
\end{array}$ & 1 & & & $\begin{array}{l}1 \\
2\end{array}$ & $\begin{array}{r}x \\
1\end{array}$ & 1 & $x$ & & & & $\begin{array}{l}x \\
x\end{array}$ & & $\mathrm{x}$ & & & & $\mathrm{x}$ \\
\hline $\begin{array}{l}\text { Brizalina bradyi } \\
\text { B. pseudopunctata } \\
\text { B. spathulata } \\
\text { B. subaenariensis } \\
\text { Buccella frigida }\end{array}$ & $\begin{array}{l}4 \\
2 \\
1\end{array}$ & $\begin{array}{r}1 \\
x \\
2 \\
2\end{array}$ & $\begin{array}{r}\mathrm{x} \\
1 \\
\mathrm{x} \\
2\end{array}$ & $\begin{array}{r}\mathrm{X} \\
3 \\
2 \\
1\end{array}$ & $\begin{array}{r}1 \\
2 \\
x \\
1\end{array}$ & $\begin{array}{r}2 \\
1 \\
X \\
2\end{array}$ & $\begin{array}{c}\mathrm{X} \\
\mathrm{X} \\
\mathrm{X} \\
4\end{array}$ & $\begin{array}{c}x \\
3\end{array}$ & $\begin{array}{l}4 \\
1 \\
5\end{array}$ & $\begin{array}{r}\mathrm{X} \\
\mathrm{X} \\
\mathrm{X} \\
2\end{array}$ & $\begin{array}{l}1 \\
1 \\
2\end{array}$ & $\begin{array}{r}\mathrm{X} \\
1 \\
1 \\
3\end{array}$ & $\begin{array}{c}\mathrm{x} \\
\mathrm{x} \\
1\end{array}$ & $\begin{array}{l}x \\
1 \\
x\end{array}$ & $\begin{array}{c}X \\
8 \\
2\end{array}$ & $\begin{array}{l}1 \\
1 \\
1\end{array}$ & $\begin{array}{r}1 \\
x \\
2 \\
1\end{array}$ \\
\hline $\begin{array}{l}\text { Bulimina aculeata } \\
\text { B. exilis } \\
\text { B. marginata } \\
\text { B. striata } \\
\text { Buliminella elegantissima }\end{array}$ & $\begin{array}{l}1 \\
1\end{array}$ & $x$ & $\mathrm{x}$ & $\begin{array}{r}\mathrm{X} \\
1 \\
2 \\
\mathrm{X}\end{array}$ & $\begin{array}{l}\mathrm{X} \\
3 \\
3\end{array}$ & $\begin{array}{r}1 \\
\times \\
3 \\
2\end{array}$ & & $\mathrm{x}$ & & $\begin{array}{l}\mathrm{X} \\
\mathrm{x}\end{array}$ & $\begin{array}{l}x \\
X \\
x\end{array}$ & $\begin{array}{l}x \\
x\end{array}$ & 1 & 1 & 1 & $\mathrm{x}$ & 3 \\
\hline $\begin{array}{l}\text { Buliminoides sp. (T) } \\
\text { Cassidulina laevigata } \\
\text { C. reniforme } \\
\text { Cibicides lobatulus } \\
\text { C. pseudoungerianus }\end{array}$ & $\stackrel{11}{1}$ & $\stackrel{9}{x}$ & ${ }^{6}$ & ले & 10 & $\begin{array}{r}10 \\
1 \\
\times\end{array}$ & $\begin{array}{r}X \\
4\end{array}$ & 3 & 6 & 2 & 10 & $x^{5}$ & 4 & $\stackrel{16}{x}$ & $\begin{array}{l}6 \\
1\end{array}$ & $\begin{array}{r}x \\
7 \\
x\end{array}$ & $\begin{array}{r}1 \\
7 \\
\times\end{array}$ \\
\hline $\begin{array}{l}\text { Dentalina hirsuta } \\
\text { D. subsoluta } \\
\text { D. subemaciata } \\
\text { Dentalina spp. } \\
\text { Discorbis cf. nitida }\end{array}$ & $x$ & & 1 & & $\begin{array}{l}x \\
x\end{array}$ & $x$ & $x$ & & 1 & 1 & 2 & $x$ & $x$ & 1 & 1 & $\begin{array}{l}\mathrm{X} \\
\mathrm{X} \\
1 \\
1\end{array}$ & $\begin{array}{l}x \\
2\end{array}$ \\
\hline $\begin{array}{l}\text { Eggerella advena } \\
\text { Elphidium excavatum f. clavata } \\
\text { E. excavatum f. excavatum } \\
\text { E. excavatum f. lidoensis } \\
\text { E. excavatum f. magna }\end{array}$ & 14 & $\stackrel{7}{x}$ & $\begin{array}{l}X \\
12 \\
X\end{array}$ & 5 & 6 & 14 & 14 & 17 & 21 & 11 & 5 & 13 & $\begin{array}{r}X \\
5\end{array}$ & 7 & $\stackrel{29}{\mathrm{X}}$ & $\begin{array}{r}15 \\
2 \\
\mathrm{X}\end{array}$ & $\begin{array}{r}20 \\
2 \\
2\end{array}$ \\
\hline $\begin{array}{l}\text { E. excavatum f. selseyensis } \\
\text { E. subarcticum } \\
\text { Eoeponides pulchella } \\
\text { Eponides bradyi } \\
\text { Epistominella exigua }\end{array}$ & $\begin{array}{l}2 \\
x \\
x \\
x\end{array}$ & $\mathrm{x}$ & 1 & $\begin{array}{l}2 \\
4 \\
5\end{array}$ & $\begin{array}{l}3 \\
1 \\
5\end{array}$ & $\begin{array}{l}3 \\
1 \\
2\end{array}$ & $\begin{array}{l}12 \\
x\end{array}$ & 30 & 9 & 13 & $\begin{array}{r}5 \\
x\end{array}$ & $\begin{array}{r}8 \\
\times\end{array}$ & $\begin{array}{l}3 \\
x \\
x\end{array}$ & $\begin{array}{r}3 \\
1 \\
1 \\
\end{array}$ & $\begin{array}{l}7 \\
1 \\
1\end{array}$ & $\begin{array}{r}\mathrm{x} \\
9 \\
\mathrm{x} \\
\mathrm{x}\end{array}$ & $\begin{array}{r}1 \\
16 \\
X \\
1\end{array}$ \\
\hline $\begin{array}{l}\text { E. takayanagii } \\
\text { E. umbonifera } \\
\text { Fissurina spp. } \\
\text { Frondiculeria sp. } \\
\text { Fursenkoina fusiformis }\end{array}$ & $\begin{array}{r}4 \\
1 \\
14\end{array}$ & 37 & 45 & $\begin{array}{r}10 \\
1 \\
15\end{array}$ & $\begin{array}{l}14 \\
\times \\
9\end{array}$ & $\begin{array}{c}8 \\
X \\
X \\
10\end{array}$ & $\begin{array}{l}43 \\
x\end{array}$ & 26 & 31 & $\begin{array}{l}40 \\
\times\end{array}$ & $\begin{array}{l}44 \\
x\end{array}$ & $\begin{array}{l}41 \\
x\end{array}$ & $\begin{array}{l}64 \\
x\end{array}$ & 38 & $\begin{array}{l}25 \\
x\end{array}$ & $\begin{array}{c}36 \\
1\end{array}$ & $\begin{array}{c}15 \\
1\end{array}$ \\
\hline $\begin{array}{l}\text { Gavelinopsis lobatulus } \\
\text { G. translucens } \\
\text { Glabratella wrightii } \\
\text { Glandulina sp. } \\
\text { Globigerinoides sp. (T) }\end{array}$ & $x$ & 4 & 4 & $x$ & $\mathrm{x}^{1}$ & $\begin{array}{l}x \\
x\end{array}$ & 3 & $\begin{array}{r}6 \\
\times\end{array}$ & 6 & 2 & $\begin{array}{r}7 \\
\times\end{array}$ & 7 & 6 & 7 & 4 & $x^{5}$ & $x^{5}$ \\
\hline
\end{tabular}

Note: $\mathrm{X}=$ less than $1 \%$. Tertiary and Cretaceous species are indicated by $\mathrm{T}$ or $\mathrm{K}$ next to the species names.

a Haynesina depressulum should be Pullenia subcarinata. 
Table 2 (continued).

\begin{tabular}{|c|c|c|c|c|c|c|c|c|c|c|c|c|c|c|c|c|c|}
\hline 117.32 & 118.81 & 120.31 & 121.81 & 123.31 & 124.81 & 126.91 & 128.41 & 129.91 & 131.41 & 132.91 & 134.91 & 136.41 & 137.91 & 139.41 & 140.91 & 142.41 & 143.91 \\
\hline $6-1$ & $6-2$ & $6-3$ & $6-4$ & $6-5$ & $6-6$ & $7-1$ & 7.2 & $7-3$ & $7-4$ & 7.5 & $7-6$ & $8-1$ & $8-2$ & $8-3$ & $8-4$ & 8-5 & $8-6$ \\
\hline 23 & 20 & 23 & 23 & 22 & 23 & 17 & 18 & 24 & 19 & 20 & 19 & 19 & 22 & 20 & 13 & 24 & 16 \\
\hline \multirow[t]{2}{*}{2016} & 2468 & 2148 & 4480 & 3936 & 1796 & 4248 & 3280 & 1004 & 2040 & 3088 & 750 & 4536 & 5000 & 4952 & 2032 & 18368 & 19648 \\
\hline & & & & & & & & & & & & 1 & & $x$ & & $x$ & \\
\hline $\mathrm{x}$ & $\mathrm{x}$ & $\mathrm{x}$ & $\begin{array}{l}x \\
x\end{array}$ & $x$ & $\underset{1}{x}$ & $\mathrm{x}$ & $\begin{array}{l}1 \\
1\end{array}$ & $\underset{1}{x}$ & $\begin{array}{l}x \\
x\end{array}$ & ${ }^{1}$ & & $\underset{1}{x}$ & & $\begin{array}{l}X \\
X \\
X\end{array}$ & 1 & $\begin{array}{l}X \\
X \\
X\end{array}$ & $\begin{array}{l}x \\
x\end{array}$ \\
\hline $\begin{array}{l}x \\
X\end{array}$ & 1 & $\mathrm{x}$ & & & & & & $x^{2}$ & $x$ & & 17 & 24 & $\underset{x}{x}$ & & & & $x$ \\
\hline $\begin{array}{l}x \\
x\end{array}$ & 2 & 11 & 12 & 6 & 4 & 4 & 4 & $\begin{array}{c}X \\
14 \\
1\end{array}$ & 6 & 6 & $\begin{array}{r}4 \\
\times\end{array}$ & 18 & 8 & 5 & 9 & 5 & 10 \\
\hline $\mathrm{x}$ & 2 & $\begin{array}{r}x \\
6\end{array}$ & $\begin{array}{c}\mathrm{X} \\
6 \\
1\end{array}$ & $\begin{array}{l}6 \\
1\end{array}$ & $\begin{array}{l}4 \\
4\end{array}$ & $\begin{array}{r}X \\
3 \\
1\end{array}$ & $\begin{array}{l}6 \\
1\end{array}$ & $x^{5}$ & $\begin{array}{l}1 \\
7 \\
2\end{array}$ & $\begin{array}{r}\mathrm{X} \\
2 \\
1\end{array}$ & 4 & $\begin{array}{l}2 \\
1\end{array}$ & $\begin{array}{l}5 \\
3\end{array}$ & $\begin{array}{l}4 \\
1\end{array}$ & $\begin{array}{l}x \\
x\end{array}$ & $\begin{array}{l}3 \\
1\end{array}$ & $\begin{array}{l}5 \\
1\end{array}$ \\
\hline & & $x$ & $x$ & $\mathrm{x}$ & $\begin{array}{l}x \\
x\end{array}$ & & & & & & & & $\mathrm{x}$ & & $\mathrm{x}$ & $x$ & \\
\hline $\begin{array}{c}28 \\
X\end{array}$ & 22 & 4 & 1 & & $\mathrm{x}^{6}$ & 2 & 5 & 9 & 3 & $\mathrm{x}^{1}$ & 18 & 4 & $x^{3}$ & 3 & 4 & $\mathrm{x}^{5}$ & 3 \\
\hline 1 & 1 & & & & & & & $x$ & & & & $\mathrm{x}$ & & & & & \\
\hline $\begin{array}{r}X \\
2\end{array}$ & 2 & 1 & $\begin{array}{l}x \\
x\end{array}$ & 1 & 1 & & 1 & $x$ & 1 & $x$ & $\underset{1}{X}$ & $\mathrm{x}$ & 1 & $\begin{array}{l}1 \\
\mathrm{x} \\
\mathrm{x}\end{array}$ & $\mathrm{x}$ & 2 & 1 \\
\hline 27 & 29 & 48 & 43 & 54 & 58 & 61 & 50 & 30 & 53 & 51 & 26 & 24 & 60 & 53 & 73 & 61 & 50 \\
\hline$x$ & 1 & 1 & 1 & 2 & 1 & $x$ & $\mathrm{x}$ & 1 & 1 & 1 & 1 & $\mathrm{x}$ & 1 & $x^{1}$ & $\mathrm{x}$ & 1 & $x$ \\
\hline 8 & 14 & 10 & 9 & 10 & 11 & 19 & 15 & 11 & 5 & 10 & 6 & 6 & 7 & 9 & 8 & 6 & 14 \\
\hline$x$ & $x^{2}$ & $x$ & 1 & 1 & $\mathrm{x}$ & $\mathrm{x}$ & 1 & $x$ & $x$ & $x$ & $x$ & & $x$ & $\mathrm{x}$ & & $x$ & \\
\hline
\end{tabular}


Table 2 (continued).

\begin{tabular}{|c|c|c|c|c|c|c|c|c|c|c|c|c|c|c|}
\hline Depth of core (m) & 145.93 & 147.43 & 148.93 & 150.43 & 151.93 & 153.43 & 155.43 & 156.93 & 158.43 & 159.43 & 161.43 & 162.93 & 185.23 & 186.73 \\
\hline Core-Section & $9-1$ & $9-2$ & $9-3$ & $9-4$ & $9-5$ & $9-6$ & $10-1$ & $10-2$ & $10-3$ & $10-4$ & $10-5$ & $10-6$ & $11-1$ & $11-2$ \\
\hline No. of species & 12 & 14 & 14 & 10 & 10 & 15 & 15 & 12 & 14 & 22 & 19 & 20 & 35 & 15 \\
\hline $\begin{array}{l}\text { Total number of } \\
\text { individuals } / 10 \mathrm{~cm}^{3}\end{array}$ & 3824 & 4936 & 5744 & 8144 & 2176 & 6688 & 4520 & 2584 & 332 & 567 & 3484 & 3840 & 2828 & 2984 \\
\hline $\begin{array}{l}\text { Astracolus crepidulus } \\
\text { Astrononion gallowayi } \\
\text { Bolivina decussata } \\
\text { B. pseudoplicata } \\
\text { B. subspinescens }\end{array}$ & & & & & & & & & 1 & & $x$ & $\mathrm{x}$ & $\begin{array}{l}x \\
x\end{array}$ & \\
\hline $\begin{array}{l}\text { Brizalina bradyi } \\
\text { B. pseudopunctata } \\
\text { B. spathulata } \\
\text { B. subaenariensis } \\
\text { Buccella frigida }\end{array}$ & $\begin{array}{l}x \\
x\end{array}$ & $\mathrm{x}$ & $\mathrm{x}$ & & $\mathrm{x}$ & $\begin{array}{l}x \\
x\end{array}$ & & & & $\begin{array}{l}\mathrm{X} \\
\mathrm{X} \\
\mathrm{X} \\
2\end{array}$ & $\begin{array}{l}x \\
x\end{array}$ & $\begin{array}{l}\mathrm{x} \\
\mathrm{x} \\
\mathrm{x}\end{array}$ & $\begin{array}{r}1 \\
1 \\
2 \\
1 \\
x\end{array}$ & 2 \\
\hline $\begin{array}{l}\text { Bulimina aculeata } \\
\text { B. exilis } \\
\text { B. marginata } \\
\text { B. striata } \\
\text { Buliminella elegantissima }\end{array}$ & 7 & 6 & 4 & 3 & 3 & 6 & 3 & 7 & 5 & $\begin{array}{r}2 \\
x\end{array}$ & $\begin{array}{r}4 \\
\times\end{array}$ & 6 & $\begin{array}{l}5 \\
2\end{array}$ & $\begin{array}{r}2 \\
x \\
\end{array}$ \\
\hline $\begin{array}{l}\text { Buliminoides sp. (T) } \\
\text { Cassidulina laevigata } \\
\text { C. reniforme } \\
\text { Cibicides lobatulus } \\
\text { C. pseudoungerianus } \\
\end{array}$ & $x^{2}$ & 1 & $\mathrm{x}$ & $x$ & & $x$ & 1 & $\mathrm{x}$ & 2 & $x$ & $x$ & $\begin{array}{l}x \\
x\end{array}$ & $\begin{array}{l}1 \\
1 \\
x\end{array}$ & $\mathrm{x}$ \\
\hline $\begin{array}{l}\text { Dentalina hirsuta } \\
\text { D. subsoluta } \\
\text { D. subemaciata } \\
\text { Dentalina spp. } \\
\text { Discorbis cf. nitida } \\
\end{array}$ & & & & & & & & & & & & $\mathrm{x}$ & $x^{2}$ & \\
\hline $\begin{array}{l}\text { Eggerella advena } \\
\text { Elphidium excavatum f. clavata } \\
\text { E. excavatum } \mathrm{f} \text {. excavatum } \\
\text { E. excavatum } \mathrm{f} \text {. lidoensis } \\
\text { E. excavatum } \mathrm{f} \text {. magna }\end{array}$ & 21 & 30 & 27 & 44 & 30 & 26 & $\begin{array}{c}30 \\
x\end{array}$ & 32 & 42 & 24 & 34 & 21 & 11 & 51 \\
\hline $\begin{array}{l}\text { E. excavatum f. selseyensis } \\
\text { E. subarcticum } \\
\text { Eoeponides pulchella } \\
\text { Eponides bradyi } \\
\text { Epistominella exigua } \\
\end{array}$ & 1 & 2 & 1 & 1 & 2 & 1 & $\begin{array}{r}5 \\
\times\end{array}$ & 1 & 2 & $\begin{array}{r}6 \\
x^{6}\end{array}$ & 6 & 2 & $\begin{array}{l}2 \\
1\end{array}$ & $\mathrm{x}^{3}$ \\
\hline $\begin{array}{l}\text { E. takayanagii } \\
\text { E. umbonifera } \\
\text { Fissurina spp. } \\
\text { Frondiculeria sp. } \\
\text { Fursenkoina fusiformis }\end{array}$ & 33 & $\begin{array}{r}28 \\
X \\
4 \\
\end{array}$ & $\begin{array}{l}37 \\
1 \\
2\end{array}$ & $\begin{array}{l}21 \\
\mathrm{x} \\
2\end{array}$ & 42 & $\begin{array}{r}27 \\
\times \\
3\end{array}$ & $\begin{array}{l}35 \\
x \\
4\end{array}$ & $\begin{array}{l}25 \\
X\end{array}$ & 13 & $\begin{array}{r}33 \\
1 \\
8\end{array}$ & $\begin{array}{l}23 \\
x \\
1\end{array}$ & $\begin{array}{l}27 \\
X^{\prime} \\
2\end{array}$ & $\begin{array}{r}24 \\
x \\
3\end{array}$ & 18 \\
\hline $\begin{array}{l}\text { Gavelinopsis lobatulus } \\
\text { G. translucens } \\
\text { Glabratella wrightii } \\
\text { Glandulina } \mathrm{sp} . \\
\text { Globigerinoides sp. (T) }\end{array}$ & & $\mathrm{x}$ & $\mathrm{x}$ & & & $\mathrm{x}$ & $\mathrm{x}$ & 1 & & 1 & $x$ & 1 & $\begin{array}{l}6 \\
6\end{array}$ & 1 \\
\hline
\end{tabular}


Table 2 (continued).

\begin{tabular}{|c|c|c|c|c|c|c|c|c|c|c|c|c|c|c|c|c|c|}
\hline Depth of core (m) & 0.2 & 0.52 & 1.02 & 19.82 & 20.32 & 22.02 & 23.32 & 24.14 & 24.72 & 25.08 & 26.02 & 26.32 & 27.72 & 28.07 & 28.74 & 59.51 & 61.01 \\
\hline Core-Section & $1-1$ & $1-1$ & $1-1$ & $2-1$ & $2-1$ & $2-2$ & $2-3$ & $2-3$ & $2-4$ & $2-4$ & $2-5$ & $2-5$ & $2-6$ & $2-6$ & $2-6$ & $4-1$ & $4-2$ \\
\hline No. of species & 34 & 17 & 21 & 45 & 42 & 43 & 19 & 14 & 14 & 21 & 22 & 21 & 20 & 21 & 23 & 38 & 36 \\
\hline $\begin{array}{l}\text { Total number of } \\
\text { individuals } / 10 \mathrm{~cm}^{3}\end{array}$ & 2344 & 900 & 790 & 1996 & 1076 & 2120 & 466 & 696 & 556 & 532 & 518 & 1172 & 604 & 478 & 1280 & 2384 & 1300 \\
\hline $\begin{array}{l}\text { Globobulimina auriculata } \\
\text { Guembelitria sp. }(\mathrm{T}-\mathrm{K}) \\
\text { Gyroidina soldanii } \\
\text { Haynesina depressulum }{ }^{\mathrm{a}} \\
\text { Heterohelix sp. (K) }\end{array}$ & $\begin{array}{l}3 \\
\mathrm{x} \\
\mathrm{x}\end{array}$ & $\mathrm{x}$ & 1 & $\begin{array}{l}\mathrm{X} \\
1 \\
3 \\
\mathrm{X} \\
\end{array}$ & $\begin{array}{r}1 \\
\mathrm{X} \\
3 \\
1\end{array}$ & $\begin{array}{r}\mathrm{x} \\
\mathrm{x} \\
\mathrm{2} \\
\mathrm{X}\end{array}$ & $\mathrm{x}$ & & $\begin{array}{l}1 \\
1\end{array}$ & & $\begin{array}{l}x \\
2\end{array}$ & 1 & & $\begin{array}{l}\mathrm{X} \\
\mathrm{X} \\
1 \\
1\end{array}$ & $\begin{array}{l}2 \\
\mathrm{X}\end{array}$ & $\begin{array}{l}\mathrm{X} \\
\mathrm{X} \\
1 \\
7 \\
\end{array}$ & $\begin{array}{r}\mathrm{X} \\
\mathrm{X} \\
1 \\
4\end{array}$ \\
\hline $\begin{array}{l}\text { Hoeglundina elegans } \\
\text { Islandiella teretis } \\
\text { Karneriella bradyi } \\
\text { Lagena spp. } \\
\text { Lenticulina gibba }\end{array}$ & $\begin{array}{l}18 \\
X\end{array}$ & $\mathrm{X}$ & $\mathrm{x}$ & $\begin{array}{c}X \\
19 \\
X \\
X\end{array}$ & $\begin{array}{c}\mathrm{X} \\
18 \\
\mathrm{X} \\
1\end{array}$ & $\begin{array}{l}\mathrm{X} \\
17 \\
\mathrm{X}\end{array}$ & 2 & $\mathrm{x}$ & 2 & 1 & 1 & $\begin{array}{l}x \\
x\end{array}$ & 1 & 1 & 1 & $\begin{array}{r}2 \\
\mathrm{x}\end{array}$ & $\mathrm{x}$ \\
\hline $\begin{array}{l}\text { Lenticulina spp. } \\
\text { Miliolinella subrotunda } \\
\text { Nodosaria sp. } \\
\text { Nonion barleeanum } \\
\text { Nonionella atlantica } \\
\end{array}$ & $\mathrm{x}$ & & & $\begin{array}{r}X \\
1\end{array}$ & $\begin{array}{l}1 \\
1\end{array}$ & $\mathrm{x}$ & & & & & & & & & & $\mathrm{x}$ & \\
\hline $\begin{array}{l}\text { N. turigida } \\
\text { Nonionellina labradorica } \\
\text { Oolina borealis } \\
\text { Oolina spp. } \\
\text { Oridorsalis umbonatus }\end{array}$ & 4 & 2 & $\mathrm{x}$ & $\begin{array}{l}3 \\
\mathrm{x} \\
\mathrm{x} \\
\mathrm{x}\end{array}$ & $\begin{array}{r}3 \\
\mathrm{x} \\
\mathrm{x}\end{array}$ & $\mathrm{x}^{4}$ & $\stackrel{5}{x}$ & 3 & 2 & 2 & 3 & 5 & 1 & 1 & $\begin{array}{l}3 \\
2\end{array}$ & $\mathrm{x}$ & $\mathrm{x}$ \\
\hline $\begin{array}{l}\text { Patellina corrugata } \\
\text { Planulina wuellerstorfi } \\
\text { Pseudopolymorphina novangliae } \\
\text { P. liqua } \\
\text { Pullenia bulloides }\end{array}$ & $\begin{array}{l}\mathrm{x} \\
\mathrm{x}\end{array}$ & & & $\begin{array}{l}x \\
1\end{array}$ & 1 & $\mathrm{x}$ & & $x$ & & $x$ & & & & & & $x$ & $x$ \\
\hline $\begin{array}{l}\text { Pyrgo williamsoni } \\
\text { Quinqueloculina seminulum } \\
\text { Q. stalkeri } \\
\text { Rectoglandulina torrida } \\
\text { Reophax arctica }\end{array}$ & $\begin{array}{r}X \\
5\end{array}$ & & $\begin{array}{r}\mathrm{X} \\
2\end{array}$ & $\begin{array}{l}\mathrm{x} \\
\mathrm{x}\end{array}$ & $x$ & $\begin{array}{r}X \\
5\end{array}$ & & 1 & & & & & $x$ & 1 & & $x$ & $x$ \\
\hline $\begin{array}{l}\text { Reusella sp. } \\
\text { Robertinoides charlottensis } \\
\text { Rosalina columbiensis } \\
\text { Sphaeroidina bulloides } \\
\text { Spirillina vivipara }\end{array}$ & & & & $\begin{array}{l}\mathrm{X} \\
\mathrm{X}\end{array}$ & $x$ & $\begin{array}{l}\mathrm{x} \\
\mathrm{x}\end{array}$ & & & & & & & & & & $x$ & $\begin{array}{l}x \\
x\end{array}$ \\
\hline $\begin{array}{l}\text { Stetsonia horvathi } \\
\text { Textularia conica } \\
T \text {. earlandi } \\
T \text {. torquata } \\
\text { Tosaia hanzawai }\end{array}$ & $\begin{array}{l}\mathrm{X} \\
\mathrm{X} \\
\mathrm{X}\end{array}$ & & & $x$ & $x$ & $\begin{array}{r}1 \\
\mathrm{X}\end{array}$ & & & & $x$ & & & & & & & \\
\hline $\begin{array}{l}\text { Trifarina fluens } \\
\text { Triloculina sp. } \\
\text { Trochammina bullata } \\
\text { T. macrescens } \\
\text { Uvigerina asperula }\end{array}$ & & & & 1 & $\begin{array}{l}x \\
x\end{array}$ & $x$ & & & & $\begin{array}{l}x \\
x\end{array}$ & & & & & 1 & $\begin{array}{l}\mathrm{X} \\
\mathrm{X}\end{array}$ & 1 \\
\hline $\begin{array}{l}\text { U. peregrina } \\
\text { Valvulinera laevigata }\end{array}$ & $\begin{array}{l}3 \\
4\end{array}$ & & & $\begin{array}{l}2 \\
2\end{array}$ & $\mathrm{x}^{2}$ & $\begin{array}{l}4 \\
1\end{array}$ & & & & $x$ & & $x$ & & $x$ & $x$ & $\begin{array}{r}\mathrm{X} \\
1\end{array}$ & $\begin{array}{r}\mathrm{X} \\
1\end{array}$ \\
\hline
\end{tabular}


Table 2 (continued).

\begin{tabular}{|c|c|c|c|c|c|c|c|c|c|c|c|c|c|c|}
\hline Depth of core (m) & 117.32 & 118.81 & 120.31 & 121.81 & 123.31 & 124.81 & 126.91 & 128.41 & 129.91 & 131.41 & 132.91 & 134.91 & 136.41 & 137.91 \\
\hline Core-Section & $6-1$ & $6-2$ & $6-3$ & $6-4$ & $6-5$ & $6-6$ & $7-1$ & $7-2$ & $7-3$ & $7-4$ & $7-5$ & $7-6$ & $8-1$ & $8-2$ \\
\hline No. of species & 23 & 20 & 23 & 23 & 22 & 23 & 17 & 18 & 24 & 19 & 20 & 19 & 19 & 22 \\
\hline $\begin{array}{l}\text { Total number of } \\
\text { individuals } / 10 \mathrm{~cm}^{3}\end{array}$ & 2016 & 2468 & 2148 & 4480 & 3936 & 1796 & 4248 & 3280 & 1004 & 2040 & 3088 & 750 & 4536 & 5000 \\
\hline $\begin{array}{l}\text { Globobulimina auriculata } \\
\text { Guembelitria sp. (T-K) } \\
\text { Gyroidina soldanii } \\
\text { Haynesina depressulum } \\
\text { Heterohelix sp. (K) }\end{array}$ & $\mathrm{x}$ & $\mathrm{x}$ & $\begin{array}{r}1 \\
\mathrm{X}\end{array}$ & & 1 & & $x$ & $\mathrm{x}$ & & $\mathrm{x}$ & $\mathrm{x}$ & $\mathrm{x}$ & 1 & $\mathrm{x}$ \\
\hline $\begin{array}{l}\text { Hoeglundina elegans } \\
\text { Islandiella teretis } \\
\text { Karreriella bradyi } \\
\text { Lagena spp. } \\
\text { Lenticulina gibba }\end{array}$ & 11 & $\begin{array}{r}3 \\
x\end{array}$ & $\begin{array}{l}9 \\
1\end{array}$ & $\begin{array}{l}13 \\
x\end{array}$ & $\begin{array}{r}6 \\
\times\end{array}$ & $\begin{array}{r}3 \\
\times\end{array}$ & $\begin{array}{r}2 \\
x\end{array}$ & $\begin{array}{r}3 \\
x\end{array}$ & 14 & $\begin{array}{l}15 \\
x\end{array}$ & $\begin{array}{l}22 \\
x\end{array}$ & $\begin{array}{l}11 \\
x\end{array}$ & $\begin{array}{l}3 \\
x\end{array}$ & $\begin{array}{l}5 \\
x \\
x\end{array}$ \\
\hline $\begin{array}{l}\text { Lenticulina spp. } \\
\text { Miliolinella subrotunda } \\
\text { Nodosaria sp. } \\
\text { Nonion barleeanum } \\
\text { Nonionella atlantica }\end{array}$ & $\mathrm{x}$ & & $\mathrm{x}$ & $\mathrm{x}$ & $\mathrm{x}$ & $\mathrm{x}$ & & & $\mathrm{x}$ & & $\mathrm{x}$ & & & \\
\hline $\begin{array}{l}\text { N. turigida } \\
\text { Nonionellina labradorica } \\
\text { Oolina borealis } \\
\text { Oolina spp. } \\
\text { Oridorsalis umbonatus }\end{array}$ & 15 & 14 & 3 & $\begin{array}{l}6 \\
x\end{array}$ & 5 & 4 & 5 & 7 & 4 & 3 & 2 & 7 & 12 & 2 \\
\hline $\begin{array}{l}\text { Patellina corrugata } \\
\text { Planulina wuellerstorfi } \\
\text { Pseudopolymorphina novangliae } \\
\text { P. liqua } \\
\text { Pullenia bulloides }\end{array}$ & $x$ & $\mathrm{x}$ & 1 & $\begin{array}{l}x \\
x\end{array}$ & $\mathrm{x}$ & & $\mathrm{x}$ & & 1 & & $x$ & & & $\mathrm{x}$ \\
\hline $\begin{array}{l}\text { Pyrgo williamsoni } \\
\text { Quinqueloculina seminulum } \\
\text { Q. stalkeri } \\
\text { Rectoglandulina torrida } \\
\text { Reophax arctica }\end{array}$ & $\begin{array}{r}1 \\
3 \\
x\end{array}$ & $\begin{array}{r}2 \\
2 \\
\mathrm{x}\end{array}$ & 1 & $\mathrm{x}$ & $\stackrel{x}{1}$ & $\begin{array}{l}x \\
x\end{array}$ & & $\begin{array}{l}1 \\
3\end{array}$ & $\begin{array}{l}\mathrm{X} \\
2\end{array}$ & & & $\begin{array}{r}\mathrm{X} \\
1 \\
\mathrm{x}\end{array}$ & & $\begin{array}{l}\mathbf{x} \\
\mathrm{x}\end{array}$ \\
\hline $\begin{array}{l}\text { Reusella sp. } \\
\text { Robertinoides charlottensis } \\
\text { Rosalina columbiensis } \\
\text { Sphaeroidina bulloides } \\
\text { Spirillina vivipara }\end{array}$ & & & $\mathrm{x}$ & & $\mathrm{x}$ & $\mathrm{x}$ & & & & & & & & \\
\hline $\begin{array}{l}\text { Stetsonia horvathi } \\
\text { Textularia conica } \\
\text { T. earlandi } \\
\text { T. torquata } \\
\text { Tosaia hanzawai }\end{array}$ & & & $\mathrm{x}$ & & & & & & & & & & & \\
\hline $\begin{array}{l}\text { Trifarina fluens } \\
\text { Triloculina sp. } \\
\text { Trochammina bullata } \\
\text { T. macrescens } \\
\text { Uvigerina asperula } \\
\end{array}$ & & & $\mathrm{x}$ & 1 & $x$ & & $x$ & $x$ & 2 & $\mathrm{x}$ & $\mathrm{x}$ & $\mathrm{x}$ & $x$ & 2 \\
\hline $\begin{array}{l}\text { U. peregrina } \\
\text { Valvulinera laevigata }\end{array}$ & & & & & & $\mathrm{x}$ & & & $\mathrm{x}$ & $\mathrm{x}$ & & & $\mathrm{x}$ & \\
\hline
\end{tabular}


Table 2 (continued).

\begin{tabular}{|c|c|c|c|c|c|c|c|c|c|c|c|c|c|c|c|c|c|}
\hline 139.41 & 140.91 & 142.41 & 143.91 & 145.93 & 147.43 & 148.93 & 150.43 & 151.93 & 153.43 & 155.43 & 156.93 & 158.43 & 159.43 & 161.43 & 162.93 & 185.23 & 186.73 \\
\hline $8-3$ & $8-4$ & $8-5$ & $8-6$ & $9-1$ & $9-2$ & 9-3 & $9-4$ & $9-5$ & $9-6$ & $10-1$ & $10-2$ & $10-3$ & $10-4$ & $10-5$ & $10-6$ & $11-1$ & $11-2$ \\
\hline 20 & 13 & 24 & 16 & 12 & 14 & 14 & 10 & 10 & 15 & 15 & 12 & 14 & 22 & 19 & 20 & 35 & 15 \\
\hline \multirow[t]{2}{*}{4952} & 2032 & 18368 & 19648 & 3824 & 4936 & 5744 & 8144 & 2176 & 6688 & 4520 & 2584 & 332 & 567 & 3484 & 3840 & 2828 & 2984 \\
\hline & $x$ & $x$ & & & & & & $\mathrm{x}$ & $x$ & & & 1 & $\begin{array}{l}x \\
x\end{array}$ & $x$ & $x$ & $\begin{array}{c}x \\
2 \\
x \\
14\end{array}$ & \\
\hline 12 & 1 & 5 & 10 & 24 & 21 & 24 & 26 & 16 & 31 & 17 & 29 & 16 & 14 & 26 & 36 & 14 & 15 \\
\hline \multirow[t]{2}{*}{1} & & $x$ & $x$ & & & & & & $x$ & $x$ & $x$ & & 1 & & $x$ & & 1 \\
\hline & & & & & & & & & & $\mathrm{x}$ & & & & & & $\begin{array}{l}X \\
1\end{array}$ & \\
\hline \multirow[t]{2}{*}{7} & $x$ & 5 & 3 & 2 & 4 & $\mathrm{x}^{1}$ & 2 & 4 & 3 & $x^{3}$ & 4 & $\begin{array}{l}7 \\
3\end{array}$ & $\mathrm{x}^{2}$ & $\begin{array}{l}x^{3} \\
x\end{array}$ & 1 & $\begin{array}{l}1 \\
\mathrm{x}\end{array}$ & 3 \\
\hline & & & & & $x$ & & & & & $x$ & & 1 & & & $\mathrm{x}$ & $x$ & \\
\hline \multirow[t]{5}{*}{$x$} & & $\begin{array}{l}x \\
x \\
x\end{array}$ & $\mathrm{x}$ & $\mathrm{x}$ & $\begin{array}{l}x \\
x\end{array}$ & 1 & $x$ & & & & $x$ & $\begin{array}{l}1 \\
4\end{array}$ & $\begin{array}{l}1 \\
x\end{array}$ & $x$ & $\begin{array}{r}1 \\
x\end{array}$ & $\underset{1}{\mathrm{X}}$ & \\
\hline & & & & & & & & & & & & & & & & & $\mathrm{x}$ \\
\hline & & & & & & & & & & & & & & & & $x$ & \\
\hline & & $\mathrm{x}$ & 1 & & & $x$ & & & & & & & & & & & \\
\hline & & $x$ & & & & & & & $x$ & & & & & & & 1 & \\
\hline
\end{tabular}



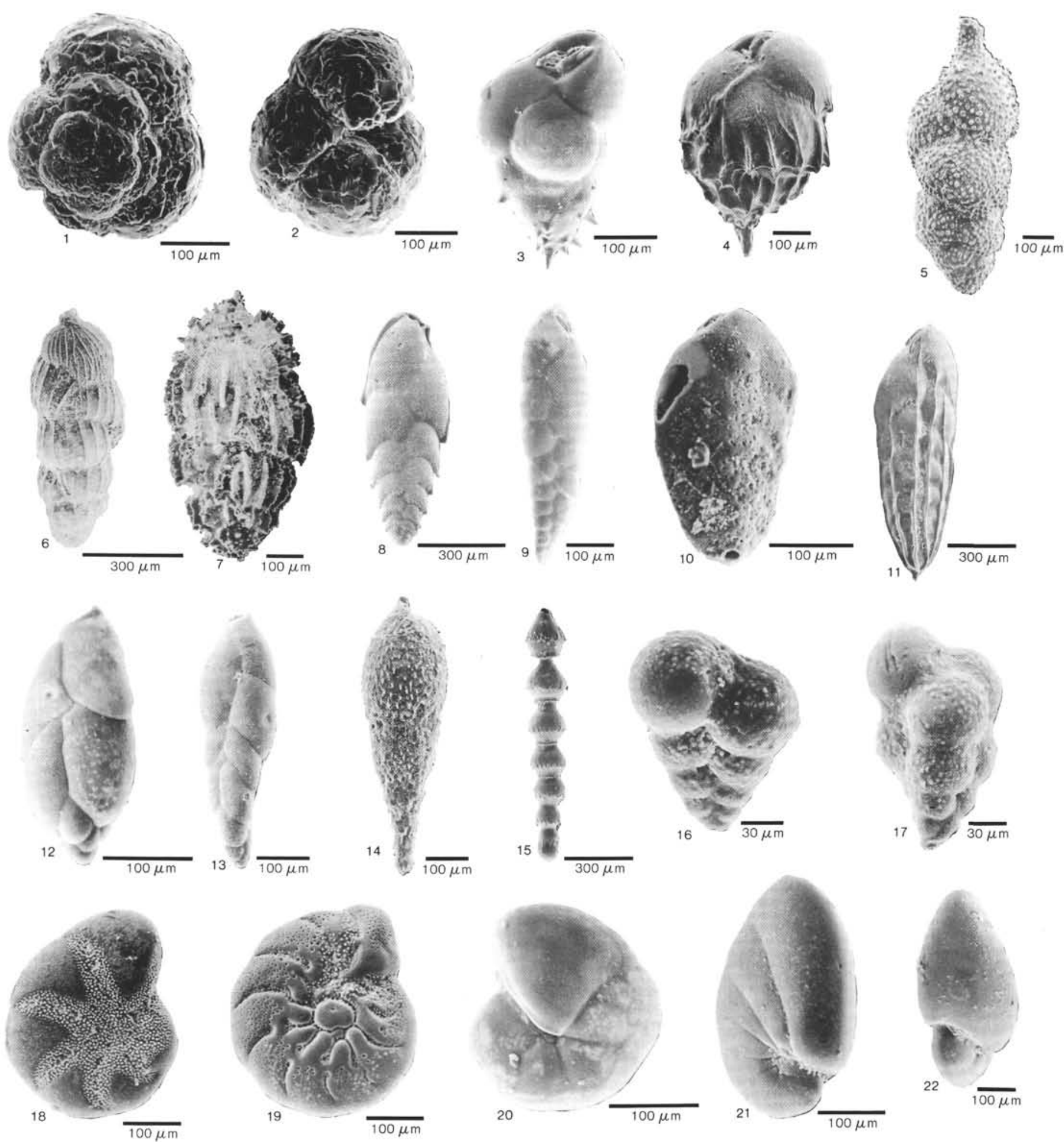

Plate 1. Benthic foraminifers. (All from Site 612 except 11, 16 and 17 from Site 613.) 1-2. Trochammina bullata, (1) dorsal view, (2) ventral view. 3. Bulimina aculeata. 4. Bulimina striata. 5. Uvigerina asperula. 6-7. Uvigerina peregrina, (6) abraded form, (7) strongly costate form. 8. Brizalina bradyi. 9. Brizalina pseudopunctata. 10. Brizalina spathulata. 11. Brizalina subaenariensis. 12-13. Fursenkoina fusiformis, (12) small typical form, (13) larger form. 14. Dentalina subsoluta. 15. Stilostomella bradyi. 16. Heterohelix sp. 17. Guembelitria sp. 18. Elphidium subarcticum. 19. Elphidium excavatum forma clavata. 20. Pullenia subcarinata. 21-22. Nonionella turigida, (21) umbilical view, (22) apertural view. 

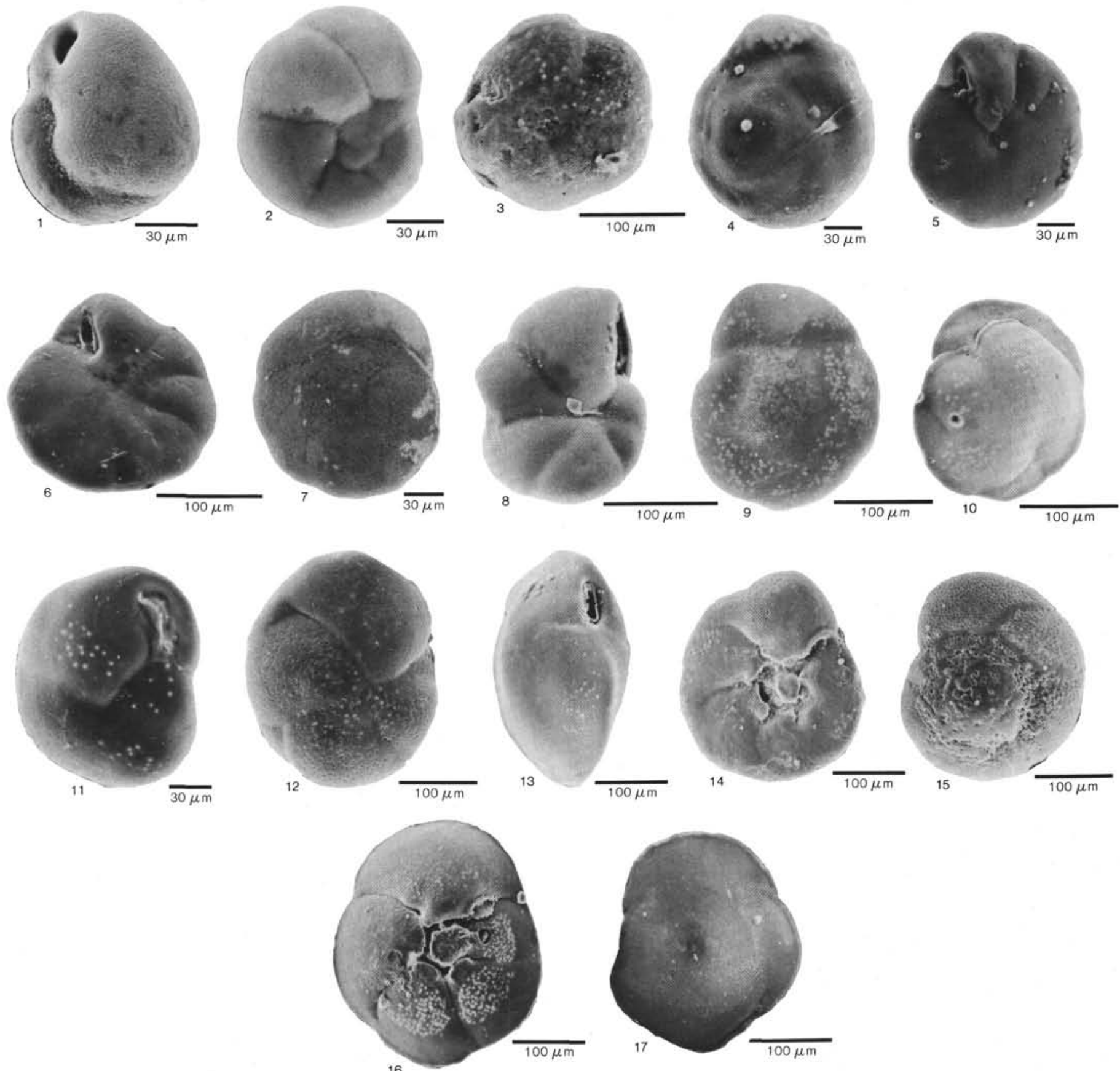

Plate 2. Benthic foraminifers. (All from Site 612 except 5, 6, 7, 14 and 15 from Site 613.) 1-2. Stetsonia horvathi,(1) ventral view, (2) dorsal view. 3-4. Eponides bradyi, (3) ventral view, (4) dorsal view. 5-7. Epistominella takayanagii, (5) ventral view of small specimen, (6) ventral view of larger specimen, (7) dorsal view. 8-9. Epistominella exigua, (8) ventral view, (9) dorsal view. 10. Cassidulina laevigata. 11-12. Cassidulina reniforme, (11) apertural view, (12) dorsal view. 13. Islandiella teretis. 14-15. Gavelinopsis translucens, (14) ventral view, (15) dorsal view. 16-17. Gavelinopsis lobatulus, (16) ventral view, (17) dorsal view. 